\title{
A biharmonic maximum principle for hyperbolic surfaces
}

\author{
By Håkan Hedenmalm, Stefan Jakobsson and Sergei Shimorin at Lund
}

\section{Introduction}

Let $\boldsymbol{\Omega}$ be a simply connected two-dimensional Riemannian manifold with a $C^{\infty}$. smooth metric $d \mathbf{s}$. We can always introduce local isothermal coordinates near any point of $\boldsymbol{\Omega}$, that is, such coordinates $(x, y)$ that the metric is represented in the form $d \mathbf{s}(x, y)^{2}=\omega(x, y)\left(d x^{2}+d y^{2}\right)$, for some positive weight function $\omega$. And since $\boldsymbol{\Omega}$ is orientable as a two-dimensional simply-connected manifold, these local isothermal coordinates can serve as conformal charts for a complex structure on $\boldsymbol{\Omega}$ (see [1], pp. 124-126). The Kœbe uniformization theorem then says that $\boldsymbol{\Omega}$ is conformally equivalent to one of the three sets: the Riemann sphere $\mathbb{S}=\mathbb{C} \cup\{\infty\}$, the complex plane $\mathbb{C}$, or the open unit disk $\mathbb{D}$. This equivalence, together with the choice of isothermal coordinates, allows us to identify $\Omega$ with one of the above three sets $\Omega$ supplied with the isothermal Riemannian metric

$$
d \mathbf{s}(z)^{2}=\omega(z)|d z|^{2}, \quad z \in \Omega \backslash\{\infty\} .
$$

Here, $\omega$ is a weight function which is strictly positive and $C^{\infty}$-smooth in $\Omega \backslash\{\infty\}$ and vanishes at infinity if $\Omega=\mathbb{S}$. The Gaussian curvature corresponding to the above isothermal metric (1.1) is given by the expression

$$
\boldsymbol{\kappa}(z)=-2 \frac{\Delta(\log \omega)(z)}{\omega(z)}, \quad z \in \Omega \backslash\{\infty\},
$$

where $\Delta$ stands for the normalized Laplacian in the plane:

$$
\Delta=\Delta_{z}=\frac{1}{4}\left(\frac{\partial^{2}}{\partial x^{2}}+\frac{\partial^{2}}{\partial y^{2}}\right), \quad z=x+i y .
$$

The (normalized) Laplace-Beltrami operator $\boldsymbol{\Delta}$ on the surface $\boldsymbol{\Omega}$ is given in terms of our choice of coordinates by the expression

$$
\Delta=\Delta_{z}=\frac{1}{\omega(z)} \Delta, \quad z \in \Omega .
$$


The Riemannian manifold $\boldsymbol{\Omega}$ is said to be hyperbolic if the Gaussian curvature is negative everywhere. For the metric (1.1), this means that $\log \omega$ should be subharmonic in $\Omega \backslash\{\infty\}$. Such functions $\omega$ are called logarithmically subharmonic. We note that this rules out the Riemann sphere as a possibility for $\Omega$, because $\log \omega$ tends to $-\infty$ at infinity, which is not possible for a subharmonic function that is not identically $-\infty$. This observation - that the sphere cannot be supplied with a hyperbolic metric - can also be easily derived from the Gauss-Bonnet formula [5], p. 417. In what follows, we deal exclusively with hyperbolic surfaces $\mathbf{\Omega}$.

The classical maximum principle for the Laplace-Beltrami operator. A real-valued function $u$ on $\boldsymbol{\Omega}$ is harmonic if $\boldsymbol{\Delta} u=0$ there, and subharmonic if $\Delta u \geqq 0$. The classical maximum principle can be given the following formulation. Let $D$ be a precompact domain in $\boldsymbol{\Omega}$, and $u$ a function continuous on the closure of $D$. We then have the implication

$$
\left.(\mathrm{MP}: \boldsymbol{\Delta}) \quad\left\{0 \leqq\left.\Delta u\right|_{D} \text { and }\left.u\right|_{\partial D} \leqq 0\right\} \Rightarrow u\right|_{D} \leqq 0 .
$$

Moreover, unless $\left.u\right|_{D}=0$, the conclusion can be sharpened to $\left.u\right|_{D}<0$.

The squared Laplace-Beltrami operator. It is natural to try to extend the maximum principle to higher order elliptic operators: we focus on the simplest example, the squared Laplace-Beltrami operator $\Delta^{2}$. In the same way that physically, the laplacian corresponds to a membrane, the bilaplacian corresponds to a plate. In view of the nature of the boundary data for the Dirichlet problem, the maximum principle we are looking for necessarily will involve two inequalities along the boundary of the subdomain $D$, one for the functions, and another for the normal derivatives. We first need some notation. A realvalued function $u$ on $\boldsymbol{\Omega}$ is biharmonic provided that $\boldsymbol{\Delta}^{2} u=0$, and sub-biharmonic if $\boldsymbol{\Delta}^{2} u \leqq 0$ (one should think of $\boldsymbol{\Delta}$ as a negative operator, which is the reason why the inequality is switched as compared with the definition of subharmonic functions). A tentative maximum principle for $\Delta^{2}$ is

$$
\left.\left(\mathrm{MP}: \boldsymbol{\Delta}^{2}\right) \quad\left\{\left.\boldsymbol{\Delta}^{2} u\right|_{D} \leqq 0,\left.u\right|_{\partial D} \leqq 0, \text { and }\left.\frac{\partial u}{\partial \boldsymbol{n}}\right|_{\partial D} \leqq 0\right\} \Rightarrow u\right|_{D} \leqq 0
$$

where the normal derivative is calculated in the interior direction, and $D$ is some simply connected precompact subdomain of $\boldsymbol{\Omega}$ with smooth boundary. The function $u$ is assumed sufficiently smooth on $\bar{D}$. The above maximum principle can be expressed in terms of properties of the biharmonic Green function $\Gamma_{D}(z, \zeta)$ on $D$, which is the solution to the boundary value problem

$$
\begin{cases}\boldsymbol{\Delta}_{z}^{2} \boldsymbol{\Gamma}_{D}(z, \zeta)=\delta_{\zeta}(z), & z \in D, \\ \boldsymbol{\Gamma}_{D}(z, \zeta)=0, & z \in \partial D, \\ \frac{\partial}{\partial \boldsymbol{n}(z)} \boldsymbol{\Gamma}_{D}(z, \zeta)=0, & z \in \partial D,\end{cases}
$$

where $\delta_{\zeta}$ stands for the unit point mass at $\zeta \in D$. After all, by Green's formula, we have the representation 
$(1.2)$

$$
\begin{aligned}
u(z)= & \int_{D} \boldsymbol{\Gamma}_{D}(z, \zeta) \boldsymbol{\Delta}^{2} u(\zeta) d \boldsymbol{\Sigma}(\zeta) \\
& +\frac{1}{2} \int_{\partial D}\left(\boldsymbol{\Delta}_{\zeta} \boldsymbol{\Gamma}_{D}(z, \zeta) \frac{\partial u}{\partial \boldsymbol{n}}(\zeta)-\frac{\partial}{\partial \boldsymbol{n}(\zeta)} \boldsymbol{\Delta}_{\zeta} \boldsymbol{\Gamma}_{D}(z, \zeta) u(\zeta)\right) d \boldsymbol{\sigma}(\zeta), \quad z \in D
\end{aligned}
$$

where $d \boldsymbol{\Sigma}$ is normalized area measure on $\boldsymbol{\Omega}$, and likewise $d \boldsymbol{\sigma}$ is normalized arc length measure. In terms of our isothermal coordinates, we have

$$
d \boldsymbol{\Sigma}(z)=\omega(z) d \Sigma(z), \quad d \boldsymbol{\sigma}(z)=\sqrt{\omega(z)} d \sigma(z),
$$

where $d \Sigma(z)=\pi^{-1} d x d y$ for $z=x+i y$, and $d \sigma(z)=|d z| /(2 \pi)$. This means that the maximum principle (MP : $\left.\Delta^{2}\right)$ is equivalent to the following three properties of the biharmonic Green function:

$$
\begin{array}{ll}
0 \leqq \Gamma_{D}(z, \zeta), & (z, \zeta) \in D \times D, \\
0 \leqq \Delta_{z} \boldsymbol{\Gamma}_{D}(z, \zeta), & (z, \zeta) \in \partial D \times D
\end{array}
$$

and

$$
\frac{\partial}{\partial \boldsymbol{n}(z)} \boldsymbol{\Delta}_{z} \boldsymbol{\Gamma}_{D}(z, \zeta) \leqq 0, \quad(z, \zeta) \in \partial D \times D
$$

A local analysis of the Green function near the boundary based on its boundary data shows that the second property (1.4) is a consequence of the first (1.3). In other words, the tentative maximum principle (MP: $\boldsymbol{\Delta}^{2}$ ) is equivalent to properties (1.3) and (1.5).

Let us for a moment look at the Euclidean situation. Around 1900, it was knownmore or less - that the maximum principle (MP : $\boldsymbol{\Delta}^{2}$ ) is valid for domains $D$ that are circular disks. The verification is based on an explicit calculation of the biharmonic Green function; for the unit disk $\mathbb{D}=\{z \in \mathbb{C}:|z|<1\}$ it is given by

$$
\Gamma(z, \zeta)=\Gamma_{\mathbb{D}}(z, \zeta)=|z-\zeta|^{2} \log \left|\frac{z-\zeta}{1-z \bar{\zeta}}\right|^{2}+\left(1-|z|^{2}\right)\left(1-|\zeta|^{2}\right)
$$

we drop the boldface symbol to indicate that we are looking at the Euclidean case. Interest in the biharmonic equation was so great around 1900 that the French Academy of Sciences offered a special prize for its solution. As a result of this initiative, Jacques Hadamard wrote the 1908 memoir on plaques élastiques encastrées ([13], pp. 515-641). There, he suggests that the maximum principle (MP : $\Delta^{2}$ ) should be valid for more general subdomains $D$ - again in the Euclidean case. In fact, he writes: "Du moins, cette proposition, comme l'inégalité $\Gamma_{A}^{B}>0$, parait incontestable pour tout contour convexe". That is, the Green function for $\Delta^{2}$ should be positive for a much larger collection of domains than the disks, including all convex regions with smooth boundary. This was, however, later shown not to be the case, by Duffin, Lœwner, and Garabedian. In fact, it follows from Paul Garabedian's work [11] that (1.4) - and, a fortiori, (1.3) - fails when $D$ is an ellipse, provided that the ratio of the major axis to the minor axis exceeds a certain critical value $\varepsilon_{0} \approx 1.5933$. Further calculations along Garabedian's lines reveal that (1.5) fails much sooner, namely when that 
ratio exceeds another critical value, $\varepsilon_{1} \approx 1.1713$. The conclusion we draw from this is that within the family of ellipses, we cannot deviate very far from circles and keep the maximum principle (MP : $\boldsymbol{\Delta}^{2}$ ). This must be due to tension in the plate, which for some reason is subdued for nearly circular domains. The intuitive feeling we get that circles are somehow special for $\Delta^{2}$ is corroborated by Charles Lœwner's work [30], where it is shown that the only coordinate transformations (suitably modified) preserving the biharmonic functions are of Mœbius type. We refer the reader to Malyshev's survey paper [31] for related results in a different direction.

We return to our case of a curved simply connected surface $\boldsymbol{\Omega}$, with negative Gaussian curvature at each point. The first question is what domains to take to generalize the circular disks which were natural in the Euclidean case. A first guess might be to consider the metric disks. However, this choice is not the right one in view of the following comments; as a matter of fact, we conjecture that the maximum principle (MP : $\left.\Delta^{2}\right)$ is generally speaking false for them. Instead, we should consider the mean value disks $D$, defined implicitly by the mean value property

$$
r^{2} h\left(z_{0}\right)=\int_{D} h(z) d \boldsymbol{\Sigma}(z)
$$

where $h$ runs over all bounded harmonic functions on $D$. The point $z_{0} \in D \subset \boldsymbol{\Omega}$ is the "center", and $r \in] 0,+\infty$ [ is the "radius" of the "disk" $D$. It is well known that in the Euclidean case, the circular disks are uniquely determined (up to addition or removal of a set of zero area measure) by the above mean value property (this is a theorem of Bernard Epstein [38]). It turns out that in our hyperbolically curved case, the analogous result holds: the mean value disk $D$ exists and is unique for each choice of $z_{0} \in D \subset \boldsymbol{\Omega}$ and $\left.r \in\right] 0,+\infty[$, provided the surface $\boldsymbol{\Omega}$ is (metrically) complete. If we consider incomplete surfaces $\boldsymbol{\Omega}$, then we need to confine the radial parameter $r$ to a maximal interval $] 0, R[$, where $R$ is such that for that value of $r, D$ touches the boundary of $\boldsymbol{\Omega}$. These results were obtained in [23], under the regularity assumption that the Riemannian metric is real-analytically smooth (that is, $\omega$ is real-analytic). It was also shown that the mean value disks $D$ have real-analytically smooth boundaries, and that they grow real-analytically in $r$. They are the result of a physical process, a Hele-Shaw flow on $\boldsymbol{\Omega}$, arising from the injection of two-dimensional Newtonian liquid into $\boldsymbol{\Omega}$ at the point $z_{0}$. The advantage with the mean value property is that modulo the constant functions, the harmonic subspace of $L^{2}(D, d \boldsymbol{\Sigma})$ then splits as a direct orthogonal sum of the analytic subspace and the antianalytic subspace. This then leads to the formula for kernel functions

$$
\mathbf{Q}_{D}(z, \zeta)=2 \operatorname{Re} \mathbf{K}_{D}(z, \zeta)-\frac{1}{r^{2}}, \quad(z, \zeta) \in D \times D
$$

where $\mathbf{Q}_{D}(z, \zeta)$ is the reproducing kernel for the harmonic subspace of $L^{2}(D, d \boldsymbol{\Sigma})$, and $\mathbf{K}_{D}(z, \zeta)$ is the reproducing kernel for the analytic subspace. The formula

$$
\boldsymbol{\Delta}_{z} \boldsymbol{\Delta}_{\zeta} \boldsymbol{\Gamma}_{D}(z, \zeta)=\delta_{0}(z-\zeta)-\mathbf{Q}_{D}(z, \zeta)
$$

which appears as the result of playing around with Green's formula, shows the relevance of the kernel $\mathbf{Q}(z, \zeta)$ for our problem. In other words: under the mean value property of $D$, the harmonic reproducing kernel has a particularly simple relation to the analytic reproducing kernel, about which it is generally easier to gather information. 
Our main result is: $\left.\boldsymbol{\Gamma}_{D}\right|_{D \times D}>0$ holds for mean value disks $D$, which corresponds to a slightly weaker maximum principle than suggested above:

$$
\left.\left(\mathrm{MP}^{\prime}: \boldsymbol{\Delta}^{2}\right) \quad\left\{\left.\boldsymbol{\Delta}^{2} u\right|_{D} \leqq 0,\left.u\right|_{\partial D}=0, \text { and }\left.\frac{\partial u}{\partial \boldsymbol{n}}\right|_{\partial D} \leqq 0\right\} \Rightarrow u\right|_{D} \leqq 0
$$

assuming $u$ is smooth on $D$. Note that it does not matter how the Gaussian curvature varies over $\boldsymbol{\Omega}$, the only thing we need to know is that it is negative everywhere. We feel that the maximum principle (MP : $\Delta^{2}$ ) should be valid under the same assumptions; so far, however, we cannot substantiate that claim.

A conformal mapping $\mathbb{D} \rightarrow D$ pulls the coordinates back to the unit disk, and the geometry of $\boldsymbol{\Omega}$ then supplies $\mathbb{D}$ with a hyperbolic geometry. In other words, we can think of $D$ as coordinatized by the unit disk. We can then think of the weight $\omega$ as defined on $\mathbb{D}$, and a simple scaling argument allows us to assume that $\omega$ is reproducing for the origin:

$$
\int_{\mathbb{D}} h(z) \omega(z) d \Sigma(z)=h(0)
$$

for all bounded harmonic functions $h$ on $\mathbb{D}$. The biharmonic operator $\Delta^{2}$ corresponds to the weighted biharmonic operator $\Delta \omega^{-1} \Delta$; we denote by $\Gamma_{\omega}$ the Green function for $\Delta \omega^{-1} \Delta$ on the unit disk $\mathbb{D}$, which corresponds to $\Gamma_{D}$. The main result-equivalent to $\left(\mathrm{MP}^{\prime}: \Delta^{2}\right)$-is the following. Note that the strong regularity assumptions on the metric have been dropped.

Theorem 1.1. Suppose $\omega$ is a logarithmically subharmonic weight on $\mathbb{D}$ which reproduces for the origin. Then $\left.\Gamma_{\omega}\right|_{\mathbb{D} \times \mathbb{D}} \geqq 0$.

The statement of the theorem is false if we keep the reproducing property but scrap the logarithmic subharmonicity: there are simple radial weights that provide counterexamples. These examples show that hyperbolic geometry lies on the "right" side of flat geometry, in the sense that for elliptic Riemannian manifolds (with positive Gaussian curvature) there is no general theorem of the above type. Also, if we instead drop the reproducing property and keep the logarithmic subharmonicity, the result is false, as is apparent from Garabedian's work on ellipses [11].

Idea of the proof of the main theorem. Let $\omega$ be a logarithmically subharmonic weight which reproduces for the origin. We can think of $\mathbb{D}$ with the Riemannian metric $d \mathbf{s}(z)=\sqrt{\omega(z)}|d z|$ as an example of a hyperbolic simply connected manifold, and call it $\boldsymbol{\Omega}$. The metric may be quite "bumpy", but an approximation procedure allows us to concentrate on the case when $\omega$ is real-analytic and strictly positive on $\overline{\mathbb{D}}$.

Let $\boldsymbol{\Gamma}=\boldsymbol{\Gamma}_{\mathbb{D}}$ be the Green function for $\Delta^{2}$ on $\mathbb{D}$, with $\boldsymbol{\Delta}=\omega^{-1} \Delta$, as before. Since $\Delta^{2} \boldsymbol{\Gamma}(\cdot, \zeta)$ is a unit point mass at $\zeta$, it follows that

$$
\Delta_{z} \boldsymbol{\Gamma}(z, \zeta)=\mathbf{G}(z, \zeta)+\mathbf{H}(z, \zeta), \quad(z, \zeta) \in \mathbb{D} \times \mathbb{D},
$$

where

$$
\mathbf{G}(z, \zeta)=\log \left|\frac{z-\zeta}{1-z \bar{\zeta}}\right|^{2}
$$


is the Green function for the laplacian, and the function $\mathbf{H}(z, \zeta)$ is harmonic in $z$. We call the kernel function $\mathbf{H}$ the harmonic compensator, because it solves the balayage problem

$$
\int_{\mathbb{D}}[\mathbf{G}(z, \zeta)+\mathbf{H}(z, \zeta)] h(z) d \mathbf{\Sigma}(z)=0, \quad \zeta \in \mathbb{D},
$$

for all bounded harmonic functions $h$ on $\mathbb{D}$. If the Green function $\Gamma$ is positive, then a local analysis near the boundary shows that $\Delta_{z} \Gamma(z, \zeta)$ is positive on $\mathbb{T} \times \mathbb{D}$, and using the harmonicity of $\mathbf{H}$ in the first variable, it follows that $\mathbf{H}$ is positive throughout $\mathbb{D} \times \mathbb{D}$. It is much less obvious that if the harmonic compensator is positive for a certain family of weight functions of the same type as $\omega$, then we can go the other way around and obtain the positivity of $\boldsymbol{\Gamma}$. This is done with the classical Duhamel principle, in the form of Hadamard's variational formula (see [13], [19]), along the Hele-Shaw flow domains $D(r)$, for $0<r \leqq 1$, as determined implicitly by the mean value property

$$
r^{2} h(0)=\int_{D(r)} h(z) d \boldsymbol{\Sigma}(z),
$$

where $h$ ranges over all bounded harmonic functions on $D(r)$.

We already mentioned that $\Gamma$ is closely connected with the harmonic kernel function Q. In terms of the harmonic compensator, the formula connecting the two reads

$$
\boldsymbol{\Delta}_{\zeta} \mathbf{H}(z, \zeta)=-\mathbf{Q}(z, \zeta)
$$

compare with Stefan Bergman's book [6], pp. 160-164. If the harmonic compensator is positive, then a local study of the behavior near $\mathbb{T} \times \mathbb{T}$ reveals that $\left.\mathbf{Q}\right|_{\mathbb{T}^{2} \mid \delta(\mathbb{T})} \leqq 0$, where $\delta(\mathbb{T})=\{(z, z): z \in \mathbb{T}\}$ denotes the diagonal. We are led to search for some kind of reverse implication. We first study the analytic reproducing kernel function $\mathbf{K}$ for the analytic subspace of $L^{2}(\mathbb{D}, d \boldsymbol{\Sigma})$, and show that $\mathbf{Q}=2 \operatorname{Re} \mathbf{K}-1$, so that the information obtained for $\mathbf{K}$ can be readily converted to information about $\mathbf{Q}$. We obtain a representation formula for $\mathbf{K}$,

$$
\mathbf{K}(z, \zeta)=\frac{1-z \bar{\zeta} \mathbf{L}(z, \zeta)}{(1-z \bar{\zeta})^{2}}, \quad(z, \zeta) \in \mathbb{D} \times \mathbb{D}
$$

where $\mathbf{L}$ is the reproducing kernel for some Hilbert space of analytic functions on $\mathbb{D}$, which we suggest to call the deficiency space for the analytic subspace of $L^{2}(\mathbb{D}, d \boldsymbol{\Sigma})$. Using this representation, we find that $\mathbf{Q}$ is negative on $\mathbb{T}^{2} \backslash \delta(\mathbb{T})$, and in fact that

$$
\mathbf{Q}(z, \zeta) \leqq-\left(\frac{1}{\omega(z)}+\frac{1}{\omega(\zeta)}\right) \frac{1}{|z-\zeta|^{2}}, \quad(z, \zeta) \in \mathbb{T} \times \mathbb{T} \backslash \delta(\mathbb{T}) .
$$

This estimate is so strong that it allows us to go backwards, to obtain the positivity of the harmonic compensator, by means of a variational technique along the weighted Hele-Shaw flow. We use the Hadamard variational method for the laplacian to write the Green function $\mathbf{G}$ as a negative integral of a product of two Poisson kernels for the flow domains $D(r)$ over $r, 0<r<1$. Noting that $\mathbf{H}(\cdot, \zeta)$ is the orthogonal harmonic projection of the function $-\mathbf{G}(\cdot, \zeta)$, we find that it suffices to show that the harmonic projection of a positive har- 
monic function on a flow region $D(r)$, with $0<r<1$, extended to vanish on $\mathbb{D} \backslash D(r)$, is positive throughout $\mathbb{D}$. This is precisely what the estimate (1.8) permits us to do.

Notation. Throughout the paper, the word positive is normally given the weakest possible sense. So, for instance, a function $f$ is positive if $0 \leqq f$ holds pointwise, and if we wish to express that $0<f$ holds everywhere, we say that the function is strictly positive. However, when we deal with individual real numbers $x$, we adhere to the usual standard and say that $x$ is positive if $0<x$. Unless explicitly stated otherwise, normal derivatives are calculated in the interior direction. A locally summable function $f$ on the curved surface $\boldsymbol{\Omega}$ is equated with a distribution by the dual action

$$
\langle\varphi, f\rangle=\int_{\mathbf{\Omega}} \varphi(z) f(z) d \boldsymbol{\Sigma}(z),
$$

for test functions $\varphi$ : compactly supported $C^{\infty}$-smooth functions on $\boldsymbol{\Omega}$. Likewise, when we are in a planar domain $\Omega$, the identification is made with respect to the corresponding dual action

$$
\langle\varphi, f\rangle=\int_{\Omega} \varphi(z) f(z) d \Sigma(z)
$$

In the above presentation, boldface letters were used to identify geometric objects, or objects that depend on the geometry; later on, we shall use the weight $\omega$ as a subscript to indicate this dependence on the geometry. So, for instance, we shall write $K_{\omega}$ instead of $\mathbf{K}$ for the analytic kernel function.

Connection with the Bergman spaces. For $0<p<+\infty$, the Bergman space $A^{p}(\mathbb{D})$ consists of all holomorphic functions $f: \mathbb{D} \rightarrow \mathbb{C}$ with bounded norm

$$
\|f\|_{A^{p}}=\left(\int_{\mathbb{D}}|f(z)|^{p} d \Sigma(z)\right)^{\frac{1}{p}}<+\infty
$$

and there is a corresponding collection of inner functions: a function $\varphi \in A^{p}(\mathbb{D})$ is inner in $A^{p}(\mathbb{D})$ if

$$
\int_{\mathbb{D}} h(z)|\varphi(z)|^{p} d \Sigma(z)=h(0),
$$

for all bounded harmonic functions $h$ on $\mathbb{D}$. These inner functions have been studied rather extensively in recent years, primarily because of their use for the factorization of functions with respect to zeros and their relevance for operator theory (see, exempli gratia, Hedenmalm [15], Duren, Khavinson, Shapiro, Sundberg [7], [8], and Aleman, Richter, Sundberg [3]; one should compare with the more classical $A^{-\infty}$ theory of Korenblum [28]). They are analogous to the classical inner functions (Blaschke products, singular inner functions, and products of the two) which play a vital rôle in the function theory of the Hardy spaces $H^{p}(\mathbb{D})$, for $0<p<+\infty$; we recall that a holomorphic function $f: \mathbb{D} \rightarrow \mathbb{C}$ is in $H^{p}(\mathbb{D})$ if

$$
\|f\|_{H^{p}}=\sup _{0<r<1}\left(\int_{\mathbb{T}}|f(r \zeta)|^{p} d \sigma(\zeta)\right)^{\frac{1}{p}}<+\infty .
$$


For an inner function $\varphi$ in $A^{p}(\mathbb{D}),|\varphi|^{p}$ is logarithmically subharmonic and reproduces for the origin, so by our main theorem, the Green function $\Gamma_{|\varphi|^{p}}$ for the weighted biharmonic operator $\Delta|\varphi|^{-p} \Delta$ is positive. As we apply this result to the Bergman spaces $A^{p}(\mathbb{D})$, we arrive at the following. Given a zero sequence $A$ in $\mathbb{D}$ for the space $A^{p}(\mathbb{D})$, let $M_{A}$ be the subspace of all functions in $A^{p}(\mathbb{D})$ that vanish at all points of $A$, with multiplicities as prescribed by the sequence. Subspaces of the type $M_{A}$ are referred to as zero-set subspaces. Let $\varphi_{A}$ be the function that maximizes $|\varphi(0)|$, given that $\varphi$ vanishes on $A$ and has norm 1 (this does not define $\varphi_{A}$ uniquely, because we can always multiply by a unimodular constant, but this is the only obstruction; if $A$ contains the origin, we need to maximize the first non-vanishing derivative at the origin). The function $\varphi_{A}$ is an inner function in $A^{p}(\mathbb{D})$, and it has no extraneous zeros; in fact, it generates $M_{A}$ as an invariant subspace [3] (see below for a definition of the term invariant subspace). Duren, Khavinson, Shapiro, and Sundberg coined the term canonical zero divisors for these functions $\varphi_{A}$. For two zero sequences $A$ and $B$, such that $A$ is contained in $B$, it follows from the main theorem that

$$
\left\|\varphi_{A} f\right\|_{A^{p}} \leqq\left\|\varphi_{B} f\right\|_{A^{p}}
$$

for all holomorphic functions $f$ on $\mathbb{D}$. This means that the canonical zero divisors are monotonic with respect to Korenblum domination along the lattice of zero-set subspaces: following Boris Korenblum [29], we say that given two functions $F$ and $G$ in $A^{p}(\mathbb{D}), G$ dominates $F$, written $F \prec G$, provided

$$
\|F q\|_{A^{p}} \leqq\|G q\|_{A^{p}}
$$

holds for all polynomials $q$. The relation $\varphi_{A} \prec \varphi_{B}$ for $A \subset B$ was conjectured by Hedenmalm in [19], [20] as well as in Problem 12.13 in the Havin-Nikolski problem book [14]. A consequence of this result is the following. We say that a closed subspace $M$ of $A^{p}(\mathbb{D})$ is invariant provided that $S f \in M$ whenever $f \in M$, where $S$ is the shift operator: $S f(z)=z f(z)$. A particularly simple collection are the zero-set subspaces, as described above. These have the property that they have index 1 (with the exception of the trivial invariant subspace $\{0\})$, meaning that the dimension of $M / S M$ is 1 . If an invariant subspace $M$ with index 1 contains a zero-set subspace, then $M$ itself is a zero-set subspace. Here the index 1 assumption is essential [17].

It is interesting to note that in the Dirichlet space, the domination relation between the corresponding functions $\varphi_{A}, \varphi_{B}$ is reversed [2], [36].

Higher dimensions. We wish to point out that the above results have been obtained in dimension $n=2$ only. It is not clear what the appropriate generalization to higher dimensional $\mathbb{R}^{n}$, for $n=3,4,5, \ldots$, should look like. A serious obstacle is that we do not have the artillery of conformal mappings any more. These problems deserve further investigation.

\section{Bergman spaces and kernel functions}

Let $D$ be a bounded domain in the complex plane $\mathbb{C}$ (a domain is a connected open set). For an area summable function $\omega: D \rightarrow[0,+\infty$ [ which is positive on a set of positive area-measure (we call $\omega$ a weight), we let $L^{2}(D, \omega)$ be the Hilbert space of complex-valued 
Borel measurable functions on $D$ which are square summable with respect to the measure $\omega d \Sigma$ : the norm is expressed by

$$
\|f\|_{\omega}=\left(\int_{D}|f|^{2} \omega d \Sigma\right)^{\frac{1}{2}}, \quad f \in L^{2}(D, \omega) .
$$

As a Hilbert space, $L^{2}(D, \omega)$ is equipped with an inner product

$$
\langle f, g\rangle_{\omega}=\int_{D} f \bar{g} \omega d \Sigma, \quad f, g \in L^{2}(D, \omega)
$$

Bergman spaces: the general setting. Let $\subseteq$ be a complex-linear vector space whose elements are continuous functions on $D$, with the property that the elements of $\subseteq$ are square summable on $D$ with respect to the measure $\omega d \Sigma$. Since elements of $L^{2}(D, \omega)$ are really equivalence classes of functions on $D$, two functions being identified if they coincide except on a null set with respect to $\omega d \Sigma$, we cannot be certain that it is possible to identify $\mathfrak{\subseteq}$ with a linear subspace of $L^{2}(D, \omega)$. This is however the case if $\omega$ is a Bergman $\mathfrak{\subseteq}$-weight function on $D$, which requires that for all $f \in \mathfrak{S}$,

$$
|f(z)| \leqq C(K)\|f\|_{\omega}, \quad z \in K
$$

where $K$ is an arbitrary compact subset of $D$, and $C(K)$ is some positive constant which depends on $K$. We also express this property by saying that the weight admits locally uniformly bounded point evaluations for functions from $\mathfrak{\Xi}$. Under this assumption, we may form the completion of $\mathfrak{S}$ with respect to the norm $\|\cdot\|_{\omega}$, which we denote by $\mathfrak{S}^{2}(D, \omega)$. The space $\mathfrak{\Xi}^{2}(D, \omega)$ consists of continuous functions on $D$, and we can regard it as a closed subspace of $L^{2}(D, \omega)$. Clearly, it gets easier for $\omega$ to be a Bergman $\subseteq$-weight function if the generating space $\mathfrak{S}$ gets smaller. If $\omega$ is a Bergman $\mathfrak{S}$-weight function, then the point evaluation functionals at points of $\Omega$ are continuous. The representation theorem for bounded linear functionals on a Hilbert space then shows that to each $\lambda \in D$, there is a unique element $K_{\omega}^{\subseteq}(\cdot, \lambda ; D)$ in $\mathfrak{\Xi}^{2}(D, \omega)$, such that

$$
f(\lambda)=\left\langle f, K_{\omega}^{\Im}(\cdot, \lambda ; D)\right\rangle_{\omega}, \quad f \in \Xi^{2}(D, \omega) .
$$

The function $K_{\omega}^{\subseteq}(z, \zeta ; D)$, with $(z, \zeta) \in D \times D$, is called the Bergman $\mathbb{\Xi}$-kernel function for the weight $\omega$ on $D$, or alternatively, the reproducing kernel for $\mathfrak{\Xi}^{2}(D, \omega)$. The space $\mathfrak{S}^{2}(D, \omega)$ is separable because $L^{2}(D, \omega)$ is, and hence it has a countable orthonormal basis $\varphi_{1}, \varphi_{2}, \varphi_{3}, \ldots$. One shows that the Bergman $\Xi$-kernel function has the representation

$$
K_{\omega}^{\Im}(z, \zeta)=\sum_{n=1}^{\infty} \varphi_{n}(z) \bar{\varphi}_{n}(\zeta), \quad(z, \zeta) \in D \times D
$$

whence it follows that the complex conjugate of $K_{\omega}^{\Im}(z, \zeta)$ equals $K_{\omega}^{\subseteq}(\zeta, z)$.

Analytic Bergman spaces. We consider $D=\mathbb{D}$, the open unit disk. When needed, the reader can easily extend the notions about to be defined to other smooth simply connected domains. The closure of the polynomials in $L^{2}(\mathbb{D}, \omega)$ is written $P^{2}(\mathbb{D}, \omega)$, and if $\omega$ is a Bergman $\mathfrak{S}$-weight function, with $\mathfrak{\subseteq}$ being the collection of all polynomials, then $P^{2}(\mathbb{D}, \omega)$ 
is a space of holomorphic functions on $\mathbb{D}$ with locally uniformly bounded point evaluations. For the weight $\omega \equiv 1$, we write $P^{2}(\mathbb{D})$ for this space, and realize that it coincides with the usual Bergman space $A^{2}(\mathbb{D})$ of square summable analytic functions. The reproducing kernel for the space $P^{2}(\mathbb{D}, \omega)$ will be denoted by $K_{\omega}(z, \zeta)$, where the variables $z, \zeta$ range over $\mathbb{D}$ (in the setting of the previous subsection, this means that in this case, we drop the superscript $\subseteq$ used to indicate the generating set of functions).

If we were to choose as spanning space $\subseteq$ all holomorphic functions on $\mathbb{D}$ which are square integrable with respect to the measure $\omega d \Sigma$, we get another collection of Bergman spaces, closer to the standard definition of the unweighted Bergman space $A^{2}(\mathbb{D})$. Provided that point evaluations are locally uniformly bounded on this space, we obtain a Hilbert space of holomorphic functions on $\mathbb{D}$, the weighted Bergman space $A^{2}(\mathbb{D}, \omega)$. Generally speaking, $P^{2}(\mathbb{D}, \omega)$ is then a subspace of $A^{2}(\mathbb{D}, \omega)$; for weights that are smooth and strictly positive up to the boundary, the two spaces coincide. Here, we focus on the polynomially generated spaces $P^{2}(\mathbb{D}, \omega)$, and mention the bigger space mainly to give the reader a more complete picture.

We need the following lemma.

Lemma 2.1. If $\omega$ is logarithmically subharmonic, then point evaluations are locally uniformly bounded $A^{2}(\mathbb{D}, \omega)$. Consequently, $A^{2}(\mathbb{D}, \omega)$ is a Hilbert space of analytic functions on $\mathbb{D}$, and so is $P^{2}(\mathbb{D}, \omega)$.

Proof. Take an interior point $z_{0} \in \mathbb{D}$, and let $r, 0<r<+\infty$, be so small that the disk $\mathbb{D}\left(z_{0}, r\right)=\left\{z \in \mathbb{C}:\left|z-z_{0}\right|<r\right\}$ is precompact in $\mathbb{D}$. For a holomorphic function $f$ on $\mathbb{D}$, the function $|f|^{2} \omega$ is subharmonic, and therefore, by the sub-mean value property,

$$
\left|f\left(z_{0}\right)\right|^{2} \omega\left(z_{0}\right) \leqq \frac{1}{r^{2}} \int_{\mathbb{D}\left(z_{0}, r\right)}|f(z)|^{2} \omega(z) d \Sigma(z) \leqq r^{-2}\|f\|_{\omega}^{2},
$$

so that

$$
\left|f\left(z_{0}\right)\right|^{2} \leqq \frac{1}{r^{2} \omega\left(z_{0}\right)}\|f\|_{\omega}^{2}
$$

Taking logarithms, we obtain

$$
\log \left|f\left(z_{0}\right)\right| \leqq \log \frac{\|f\|_{\omega}}{r}+\frac{1}{2} \log \frac{1}{\omega\left(z_{0}\right)}
$$

The left hand side is subharmonic, so that we can get the estimate

$$
\log \left|f\left(z_{0}\right)\right| \leqq \log \frac{\|f\|_{\omega}}{r}+\frac{1}{2 r} \int_{\partial \mathbb{D}\left(z_{0}, r\right)} \log \frac{1}{\omega(z)} d \sigma(z),
$$

and since $\log \omega$ is subharmonic, it is integrable on compact circles in $\mathbb{D}$ such as $\partial \mathbb{D}\left(z_{0}, r\right)$. Introducing the Poisson kernel in these calculations allows us to get a uniform estimate on compact subsets, which does it. 
Harmonic Bergman spaces. So far we only considered Bergman spaces of holomorphic functions. We also need Bergman spaces of harmonic functions.

If we let the generating space $\mathfrak{\subseteq}$ equal the space $H P$ of harmonic polynomials, which are functions of the type $p+\bar{q}$, where $p$ and $q$ are polynomials, we get the space $H P^{2}(\mathbb{D}, \omega)$, which is a Hilbert space of harmonic functions on $\mathbb{D}$, provided the weight $\omega$ allows for locally uniformly bounded point evaluations. We call it the harmonic polynomial Bergman space with weight $\omega$ on $\mathbb{D}$. The reproducing kernel function for it is written $Q_{\omega}(z, \zeta)$, and is sometimes referred to as the harmonic polynomial Bergman kernel with weight $\omega$. As for the analytic Bergman spaces, there is a bigger space of all harmonic functions in $L^{2}(\mathbb{D}, \omega)$; however, we shall not need it.

Logarithmically subharmonic reproducing weights on the unit disk. Let $\omega$ be a logarithmically subharmonic area summable weight $\omega$ on $\mathbb{D}$, which is reproducing for the origin, in the sense of the introduction:

$$
h(0)=\int_{\mathbb{D}} h(z) \omega(z) d \Sigma(z)
$$

holds for all bounded harmonic functions $h$ on $\mathbb{D}$.

The following assertion is known, but we do not have a reference. The first result of this type can be found in [15].

Lemma 2.2. For the above class of weights $\omega$, we have the following growth control:

$$
\omega(z) \leqq\left(1-|z|^{2}\right)^{-1}, \quad z \in \mathbb{D} .
$$

Proof. To see that this estimate is valid, one can proceed as follows. As in [15], one obtains the Carleson measure type condition

$$
\int_{\mathbb{D}}|f|^{2} \omega d \Sigma \leqq\|f\|_{H^{2}}, \quad f \in H^{2}(\mathbb{D})
$$

Consider for $\zeta \in \mathbb{D}$ the Mœbius automorphism of the disk

$$
\phi_{\zeta}(z)=\frac{\zeta-z}{1-z \bar{\zeta}}, \quad z \in \mathbb{D},
$$

and note that the above inequality becomes

$$
\int_{\mathbb{D}}\left|f \circ \phi_{\zeta}\right|^{2} \omega \circ \phi_{\zeta}\left|\phi_{\zeta}^{\prime}\right|^{2} d \Sigma \leqq\|f\|_{H^{2}}, \quad f \in H^{2}(\mathbb{D}) .
$$

By choosing

$$
f(z)=\frac{\left(1-|\zeta|^{2}\right)^{\frac{1}{2}}}{1-z \bar{\zeta}}
$$


we obtain from the sub-mean value property that

$$
\left(1-|\zeta|^{2}\right) \omega(\zeta) \leqq\left(1-|\zeta|^{2}\right) \int_{\mathbb{D}} \frac{\omega \circ \phi_{\zeta}(z)\left|\phi_{\zeta}^{\prime}(z)\right|^{2}}{\left|1-\phi_{\zeta}(z) \bar{\zeta}\right|^{2}} d \Sigma(z) \leqq 1
$$

from which the assertion is immediate.

Proposition 2.3. Under the above assumptions on $\omega, H P^{2}(\mathbb{D}, \omega)$ is a Hilbert space of harmonic functions on $\mathbb{D}$, with locally uniformly bounded point evaluations. Denote by $P_{0}^{2}(\mathbb{D}, \omega)$ the subspace of $P^{2}(\mathbb{D}, \omega)$ consisting of those functions that vanish at the origin, and by $\bar{P}_{0}^{2}(\mathbb{D}, \omega)$ its image under complex conjugation. Then the harmonic space splits

$$
H P^{2}(\mathbb{D}, \omega)=P^{2}(\mathbb{D}, \omega) \oplus \bar{P}_{0}^{2}(\mathbb{D}, \omega),
$$

the two subspaces on the right hand side being orthogonal. As a consequence, the kernel function for $\mathrm{HP}^{2}(\mathbb{D}, \omega)$ has the form

$$
Q_{\omega}(z, \zeta)=2 \operatorname{Re} K_{\omega}(z, \zeta)-1, \quad(z, \zeta) \in \mathbb{D}^{2}
$$

Proof. Let $p, q$ be polynomials. If $q(0)=0$, then by the reproducing property of $\omega$,

$$
\langle p, \bar{q}\rangle_{\omega}=\int_{\mathbb{D}} p(z) q(z) \omega(z) d \Sigma(z)=0,
$$

and hence $P^{2}(\mathbb{D}, \omega)$ and $\bar{P}_{0}^{2}(\mathbb{D}, \omega)$ are perpendicular with respect to the inner product of $H P^{2}(\mathbb{D}, \omega)$. Each harmonic polynomial can be written in the form $p+\bar{q}$. By the Pythagorean theorem,

$$
\|p+\bar{q}\|_{\omega}^{2}=\|p\|_{\omega}^{2}+\|q\|_{\omega}^{2}
$$

If we take a Cauchy sequence of harmonic polynomials $p_{j}+\bar{q}_{j}\left(\right.$ with $\left.q_{j}(0)=0\right)$ with respect to the norm $\|\cdot\|_{\omega}$, then by the above, $p_{j}$ is a Cauchy sequence in $P^{2}(\mathbb{D}, \omega)$, and $q_{j}$ a Cauchy sequence in $P_{0}^{2}(\mathbb{D}, \omega)$. In view of Lemma 2.1 , there are elements $f \in P^{2}(\mathbb{D}, \omega)$ and $g$ in $P_{0}^{2}(\mathbb{D}, \omega)$, holomorphic in the disk $\mathbb{D}$, such that $p_{j} \rightarrow f$ and $q_{j} \rightarrow g$. The limit function $h=f+\bar{g}$ is then harmonic in $\mathbb{D}$, and we have

$$
\|h\|_{\omega}^{2}=\|f+\bar{g}\|_{\omega}^{2}=\|f\|_{\omega}^{2}+\|g\|_{\omega}^{2} .
$$

The local boundedness of point evaluations now follows from Lemma 2.1.

The reproducing kernel for $P^{2}(\mathbb{D}, \omega)$ is $K_{\omega}$, and for $\bar{P}_{0}^{2}(\mathbb{D}, \omega)$ it is $\bar{K}_{\omega}-1$. It follows from the above direct sum decomposition that $Q_{\omega}$ is the sum of these two kernels.

\section{Green functions for weighted biharmonic operators}

Smooth weights. Let $D$ be a simply connected bounded domain in $\mathbb{C}$ with $C^{\infty}$-smooth Jordan boundary. Also, let $v$ be strictly positive and $C^{\infty}$-smooth on the closure $\bar{D}$. We then 
define the Green function $\Gamma_{v, D}$ for the biharmonic operator $\Delta v^{-1} \Delta$ in the following way. For fixed $\zeta \in D$, it solves the boundary value problem

$$
\begin{aligned}
\Delta_{z} v(z)^{-1} \Delta_{z} \Gamma_{v, D}(z, \zeta) & =\delta_{\zeta}(z), & & z \in D, \\
\Gamma_{v, D}(z, \zeta) & =0, & & z \in \partial D, \\
\partial_{n(z)} \Gamma_{v, D}(z, \zeta) & =0, & & z \in \partial D,
\end{aligned}
$$

where the normal derivative is taken in the interior direction. The symbol $\delta_{\zeta}$ denotes a unit point mass at the point $\zeta \in D$. The laplacian $\Delta$ has the factorization

$$
\Delta_{z}=\frac{\partial^{2}}{\partial z \partial \bar{z}}
$$

where $(z=x+i y)$

$$
\frac{\partial}{\partial z}=\frac{1}{2}\left(\frac{\partial}{\partial x}-i \frac{\partial}{\partial y}\right), \quad \frac{\partial}{\partial \bar{z}}=\frac{1}{2}\left(\frac{\partial}{\partial x}+i \frac{\partial}{\partial y}\right)
$$

are the usual Wirtinger derivatives. We shall also use the space-saving notation $\partial_{z}$ and $\bar{\partial}_{z}$ for these operators. The weighted biharmonic operators $\Delta v^{-1} \Delta$ and the associated Green functions seem to have been considered for the first time by Paul Garabedian [11]. When we apply a laplacian to $\Gamma_{v, D}$, we should get the weight $v$ times the Green function $G_{D}$ for the laplacian plus a harmonic function, that is,

$$
\Delta_{z} \Gamma_{v, D}(z, \zeta)=v(z)\left[G_{D}(z, \zeta)+H_{v, D}(z, \zeta)\right], \quad(z, \zeta) \in D \times D
$$

where $H_{v, D}(z, \zeta)$ is harmonic in the $z$ variable. Let $\phi$ be a $C^{2}$ function on the closure of $D$. Applying Green's formula, we see that the zero Dirichlet boundary conditions on $\Gamma_{v}$ translate into the requirement that

$$
\int_{D} \Delta_{z} \Gamma_{v, D}(z, \zeta) \phi(z) d \Sigma(z)=\int_{D} \Gamma_{v, D}(z, \zeta) \Delta_{z} \phi(z) d \Sigma(z)
$$

By applying this identity to $\phi=h$, where $h$ is harmonic, we obtain

$$
\int_{D} h(z)\left[G_{D}(z, \zeta)+H_{v, D}(z, \zeta)\right] v(z) d \Sigma(z)=0
$$

and by an approximation argument we have this for all bounded harmonic functions $h$ on $D$. This is the balayage problem mentioned in the introduction. Since the bounded functions are dense in $H L^{2}(D, v)$, the closed subspace of $L^{2}(D, v)$ consisting of functions harmonic on $D$, it follows that $H_{v}(\cdot, \zeta)$ equals the orthogonal projection of the function $-G_{D}(\cdot, \zeta)$ to $H L^{2}(D, v)$ in the space $L^{2}(D, v)$. We shall call the kernel $H_{v, D}$ the harmonic compensator. Due to the smoothness assumption on the weight $v$ and of the simply connected domain $D$, the harmonic polynomials are dense in $H^{2}(D, v)$, so that the latter equals $H P^{2}(D, v)$, a space we encountered earlier. We write the above relationship as $H_{v, D}=-Q_{v, D} M_{v} G_{D}\left(M_{v}\right.$ is the operator of multiplication by $\left.v\right)$, or, written out more explicitly, 


$$
H_{v, D}(z, \zeta)=-\int_{D} Q_{v, D}(z, \xi) G_{D}(\xi, \zeta) v(\xi) d \Sigma(\xi),
$$

where as before, $Q_{v, D}$ is the reproducing kernel for $H L^{2}(D, v)$. Here, we think of a kernel $T(z, \zeta)$ as having an operator $T$ associated to it in the fashion

$$
T f(z)=\int_{D} T(z, \zeta) f(\zeta) d \Sigma(\zeta),
$$

whenever the integral converges. In principle, the operator also determines the kernel, for we obtain the kernel by applying the operator to a unit point mass at an interior point (not an element of the space, but it can be approximated by such). Because of the boundary conditions, the Green function can be recovered through the formula

$$
\Gamma_{v, D}(z, \zeta)=\int_{D} G_{D}(z, \xi)\left[G_{D}(\xi, \zeta)+H_{v, D}(\xi, \zeta)\right] v(\xi) d \Sigma(\xi) .
$$

The operators $Q_{v, D}$ and $G_{D}$ are self-adjoint, so that taking adjoints, we have the identity $H_{v, D}^{*}=-G_{D} M_{v} Q_{v, D}$. We note that the kernel for the operator $H_{v, D}^{*}$ is $H_{v, D}^{*}(z, \zeta)=H_{v, D}(\zeta, z)$. The function $H_{v, D}(z, \cdot)$ then solves Poisson's equation with data $-v Q_{v, D}(\cdot, z)$. The kernel $H_{v, D}(z, \zeta)$ is harmonic in the $z$ variable, and for $z \in \partial D$, it solves the boundary value problem (compare with [19]; $\partial_{n}$ is a condensed notation for the interior normal derivative)

$$
\begin{aligned}
\Delta_{\zeta} v(\zeta)^{-1} \Delta_{\zeta} H_{v, D}(z, \zeta) & =0, & & \zeta \in D, \\
H_{v, D}(z, \zeta) & =0, & & \zeta \in \partial D, \\
\partial_{n(\zeta)} H_{v, D}(z, \zeta) & =2 \delta_{z}(\zeta), & & \zeta \in \partial D .
\end{aligned}
$$

Smoothness properties of kernels. As above, let $D$ be finitely connected with $C^{\infty}$ smooth boundary, and the weight $v$ be $C^{\infty}$-smooth on $\bar{D}$, and strictly positive there. For a subset $E$ of the complex plane $\mathbb{C}$, let $\delta(E)=\left\{(z, z) \in \mathbb{C}^{2}: z \in E\right\}$ be the corresponding diagonal set. In particular, we shall be concerned with the diagonal $\delta(\bar{D})$, the interior diagonal $\delta(D)$, and the boundary diagonal $\delta(\partial D)$. Then, by an elliptic regularity theorem of Louis Nirenberg [34], which says that we have $C^{\infty}$-smooth solutions locally if the data are that smooth, the kernels $G$ and $\Gamma_{v}$ are $C^{\infty}$-smooth on $(\bar{D} \times \bar{D}) \backslash \delta(\bar{D})$. It follows that the kernels $H_{v, D}$ and $Q_{v, D}$ are $C^{\infty}$-smooth on $(\bar{D} \times \bar{D}) \backslash \delta(\partial D)$.

Let us for the moment replace $C^{\infty}$-smoothness with $C^{\omega}$-smoothness-realanalyticity-everywhere above (so that the boundary $\partial D$ is real-analytic, and the weight $v$ is real-analytic on $\bar{D}$ ). Another elliptic regularity theorem, this time due to Morrey and Nirenberg [32], then states that locally, solutions are $C^{\omega}$-smooth if the data have that degree of regularity. We apply it to our Green functions, to get that the kernels $G$ and $\Gamma_{v}$ are $C^{\omega}$-smooth on $(\bar{D} \times \bar{D}) \backslash \delta(\bar{D})$, and that as before, it follows that the kernels $H_{v, D}$ and $Q_{v, D}$ are $C^{\omega}$-smooth on $(\bar{D} \times \bar{D}) \backslash \delta(\partial D)$.

If we instead consider the complex elliptic second order operator $\bar{\partial}_{z} v^{-1} \partial_{z}$, we have the same regularity theory. The associated Green function $G_{v, D}$ (with zero Dirichlet boundary data) was considered by Garabedian in [11]. He obtained the identity

$$
\partial_{z} \bar{\partial}_{\zeta} G_{v, D}(z, \zeta)=v(z) v(\zeta) K_{v, D}(z, \zeta), \quad(z, \zeta) \in D^{2} \backslash \delta(D),
$$


which carries over the regularity of $G_{v, D}$ to the analytic reproducing kernel $K_{v, D}$. Suppose we are in the $C^{\infty}$-smooth situation, which means that the weight $v$ is assumed $C^{\infty}$-smooth and strictly positive on $\bar{D}$, and the Jordan curve $\partial D$ is assumed $C^{\infty}$-smooth, too. Then the regularity of $G_{v, D}$ shows that $K_{v, D}$ is $C^{\infty}$-smooth on $(\bar{D} \times \bar{D}) \backslash \delta(\partial D)$. Similarly, in the real-analytic situation, we have the following: if $v$ is assumed $C^{\omega}$-smooth and strictly positive on $\bar{D}$, and $\partial D$ is $C^{\omega}$-smooth as well, it follows that the kernel $K_{v, D}$ is $C^{\omega}$-smooth on $(\bar{D} \times \bar{D}) \backslash \delta(\partial D)$. This latter fact was mentioned and used in [7].

Some consequences of the positivity of $\Gamma_{\boldsymbol{v}, \boldsymbol{D}}$. Let us assume $D$ and $v$ are as above, and that we know that $\Gamma_{v, D}$ is positive on $D \times D$. For fixed $\zeta \in D$, the function $\Gamma_{v}(\cdot, \zeta)$ vanishes together with its normal derivative along $\partial D$, so for it to be positive in the interior it must have a positive second normal derivative, that is, $0 \leqq \Delta \Gamma_{v, D}(\cdot, \zeta)$ on $\partial D$. By $(3.1)$, this means that $H_{v, D}(\cdot, \zeta)$ is positive on $\partial D$, so by harmonicity, we even get that $H_{v, D}(\cdot, \zeta)$ is positive on $D$. By a similar type of argument applied to the second coordinate, we see that the harmonic kernel function $Q_{v, D}(z, \zeta)$ is negative for $(z, \zeta) \in(\partial D \times \partial D) \backslash \delta(\partial D)$. This consequence was observed by Garabedian [11], and in fact, it was how he showed that the biharmonic Green function fails to be positive for sufficiently eccentric ellipses. We use it with a different purpose in mind: we need to know what properties to look for in the harmonic kernel function $Q_{v, D}$ to be able to prove that we have a positive Green function $\Gamma_{v, D}$.

More general weights. What if the weight is less smooth than assumed previously? For instance, let us say that we merely know that $v$ is positive and area-summable on the $C^{\infty}$-smooth Jordan domain $D$. It is fairly clear that some additional requirement is needed to be able to define the biharmonic Green function $\Gamma_{v, D}$, in view of the formulæ (3.1) and (3.3), which suggests that the existence of $\Gamma_{v, D}$ entails that some type of harmonic Bergman space then becomes well-defined for the weight $v$. Actually, we can simply use the formulæ (3.3) and (3.4) to define the weighted biharmonic Green function $\Gamma_{v, D}$, provided that the weight is such that the harmonic polynomial Bergman space is well-defined, so that we can access its reproducing kernel $Q_{v, D}$.

We formalize this in a definition.

Definition 3.1. Let $v$ be a weight on $D$, such that the point evaluations are locally uniformly bounded on $D$ for the space and let $G_{D}$ stand for the Green function for the laplacian $\Delta$ on $D$. The harmonic compensator is the function

$$
H_{v, D}(z, \zeta)=-\int_{D} Q_{v, D}(z, \xi) G_{D}(\xi, \zeta) v(\xi) d \Sigma(\xi),
$$

and the weighted biharmonic Green function is given by

$$
\Gamma_{v, D}(z, \zeta)=\int_{D} G_{D}(z, \xi)\left[G_{D}(\xi, \zeta)+H_{v, D}(\xi, \zeta)\right] v(\xi) d \Sigma(\xi)
$$

\section{The smoothing of weights}

Here we show how to obtain the following approximation result.

Theorem 4.1. Let $\omega$ be logarithmically subharmonic weight on $\mathbb{D}$ which is reproducing for the origin. Then, for each $\varepsilon, 0<\varepsilon<+\infty$, there is another logarithmically subharmonic 
reproducing weight $\tilde{\omega}$ which is real-analytic on the closed disk $\overline{\mathbb{D}}$ and strictly positive there, such that

$$
\int_{\mathbb{D}}|\omega(z)-\tilde{\omega}(z)| d \Sigma(z)<\varepsilon
$$

The local smoothing of weights. Let aut $(\mathbb{D})$ denote the automorphism group of $\mathbb{D}$, which consists of all conformal mappings of $\mathbb{D}$ onto itself. If we let $\phi$ be an element of aut $(\mathbb{D})$, then we can find $\alpha, \beta \in \mathbb{T}$ and $r \in[0,1[$ such that

$$
\phi=R_{\alpha} \circ \phi_{r} \circ R_{\beta}
$$

where $R_{\alpha}(z)=\alpha z$ and $R_{\beta}(z)=\beta z$ are rotations, and

$$
\phi_{r}(z)=\frac{r-z}{1-r z}
$$

is a kind of reflexion. This decomposition is unique for $r \neq 0$, and for $r=0$, the mapping $\phi$ is a rotation, and only the product $\alpha \beta$ can be determined. For complex $\lambda \in \mathbb{D}$, set

$$
\phi_{\lambda}(z)=\frac{\lambda-z}{1-\bar{\lambda} z}
$$

which decomposes into

$$
\phi_{\lambda}=R_{\alpha} \circ \phi_{r} \circ R_{\bar{\alpha}}
$$

provided that $\lambda=r \alpha$, with $0 \leqq r<1$ and $\alpha \in \mathbb{T}$. So we can write a general $\phi \in \operatorname{aut}(\mathbb{D})$ as $\phi=R_{\beta} \circ \phi_{\lambda}$, with $\beta \in \mathbb{T}$ and $\lambda \in \mathbb{D}$. In this decomposition, both $\beta$ and $\lambda$ are uniquely determined. We can then identify aut $(\mathbb{D})$ with the set $\mathbb{T} \times \mathbb{D}$, which can be visualized as a subset of $\mathbb{R}^{4}$. Thinking of $\mathbb{R}^{4}$ as a subset of the complex four-dimensional space $\mathbb{C}^{4}$, we can define real-analytic functions on $\mathbb{T} \times \mathbb{D}$ as those that extend holomorphically to some open subset of $\mathbb{C}^{4}$ containing $\mathbb{T} \times \mathbb{D}$. We can also think of another complex structure: $\mathbb{T} \times \mathbb{D} \subset \mathbb{C}^{3}$, viewing $\mathbb{T}$ as a subset of $\mathbb{C}$, and $\mathbb{D}$ as a subset of $\mathbb{R}^{2} \subset \mathbb{C}^{2}$. This complex structure also gives rise to a class of real-analytic functions. Fortunately, the two different complex structures induce the same class of real-analytic functions.

There is a left and right invariant Haar measure on aut $(\mathbb{D})$, which in terms of the representation (4.1) takes the form

$$
d \phi=\frac{2 r d r}{\left(1-r^{2}\right)^{2}} d \sigma(\alpha) d \sigma(\beta),
$$

and in terms of the representation $\phi=R_{\beta} \circ \phi_{\lambda}$, with $(\beta, \lambda) \in \mathbb{T} \times \mathbb{D}$, it becomes

$$
d \phi=\frac{d \Sigma(\lambda)}{\left(1-|\lambda|^{2}\right)^{2}} d \sigma(\beta) .
$$


Let $\Phi: \operatorname{aut}(\mathbb{D}) \rightarrow] 0,+\infty[$ be a real-analytic function of the product form

$$
\Phi(\phi)=\Phi_{1}(\beta) \Phi_{2}(\lambda), \quad \phi=R_{\beta} \circ \phi_{\lambda}
$$

where

$$
\Phi_{2}(\lambda)=(N-1)\left(1-|\lambda|^{2}\right)^{N}, \quad \lambda \in \mathbb{D},
$$

for some integer $N=2,3,4, \ldots$, which has integral

$$
\int_{\mathbb{D}} \Phi_{2}(\lambda) \frac{d \Sigma(\lambda)}{\left(1-|\lambda|^{2}\right)^{2}}=1,
$$

and $\left.\Phi_{1}: \mathbb{T} \rightarrow\right] 0,+\infty[$ is some real-analytic function with integral

$$
\int_{\mathbb{T}} \Phi_{1}(\beta) d \sigma(\beta)=1
$$

For instance, we can take

$$
\Phi_{1}(\beta)=\frac{1-\varrho^{2}}{|1+\varrho \beta|^{2}}, \quad \beta \in \mathbb{T},
$$

for some real parameter $\varrho$ with $0<\varrho<1$. We shall now see that

$$
\int_{\operatorname{aut}(\mathbb{D})} \Phi(\phi) h \circ \phi^{-1}(0) d \phi=h(0)
$$

for all bounded harmonic functions $h$ on $\mathbb{D}$. For $\phi=R_{\beta} \circ \phi_{\lambda}$, we have $\phi^{-1}=\phi_{\lambda} \circ R_{\bar{\beta}}$, so that $\phi^{-1}(0)=\lambda$. It follows that the left hand side of (4.4) assumes the form

$$
\begin{aligned}
\int_{\mathbb{T} \times \mathbb{D}} \Phi_{1}(\beta) \Phi_{2}(\lambda) h(\lambda) d \sigma(\beta) \frac{d \Sigma(\lambda)}{\left(1-|\lambda|^{2}\right)^{2}} \\
\quad=\int_{\mathbb{T}} \Phi_{1}(\beta) d \sigma(\beta) \int_{\mathbb{D}}(N-1)\left(1-|\lambda|^{2}\right)^{N-2} h(\lambda) d \Sigma(\lambda)=h(0),
\end{aligned}
$$

for all bounded harmonic functions $h$ on $\mathbb{D}$, as claimed, if we use the mean value property. We shall use the function $\Phi$ to regularize $\omega$ : consider the function

$$
\omega_{\Phi}(z)=\int_{\operatorname{aut}(\mathbb{D})} \Phi(\phi) \omega \circ \phi(z)\left|\phi^{\prime}(z)\right|^{2} d \phi .
$$

It is strictly positive on $\mathbb{D}$, because with the given choice of the smoothing function $\Phi$, the only way for $\omega_{\Phi}$ to vanish at a point $z \in \mathbb{D}$ would be that $\omega \circ \phi(z)=0$ for almost all $\phi$ in $\operatorname{aut}(\mathbb{D})$, which never happens, given the assumptions on $\omega$. Just as $\omega$, the function $\omega_{\Phi}$ is logarithmically subharmonic, because each individual function $\omega \circ \phi\left|\phi^{\prime}\right|^{2}$ occurring in the integral is, and because the logarithmically subharmonic functions form a cone. It is representing as well, as a computation shows: 


$$
\begin{aligned}
\int_{\mathbb{D}} h(z) \omega_{\Phi}(z) d \Sigma(z) & =\int_{\operatorname{aut}(\mathbb{D})} \Phi(\phi) \int_{\mathbb{D}} h(z) \omega \circ \phi(z)\left|\phi^{\prime}(z)\right|^{2} d \Sigma(z) d \phi \\
& =\int_{\operatorname{aut}(\mathbb{D})} \Phi(\phi) \int_{\mathbb{D}} h \circ \phi^{-1}(z) \omega(z) d \Sigma(z) d \phi \\
& =\int_{\operatorname{aut}(\mathbb{D})} \Phi(\phi) h \circ \phi^{-1}(0) d \phi=h(0),
\end{aligned}
$$

for all bounded harmonic functions $h$ on $\mathbb{D}$, where we use (4.4). A shift of variables yields the alternative representation

$$
\omega_{\Phi}(z)=\left(1-|z|^{2}\right)^{-2} \int_{\operatorname{aut}(\mathbb{D})} \Phi\left(\phi \circ \phi_{z}\right) \omega \circ \phi(0)\left|\phi^{\prime}(0)\right|^{2} d \phi
$$

This is a mean of the various functions $\Phi\left(\phi \circ \phi_{z}\right)$, taken over the variable $\phi$, because

$$
\begin{aligned}
\int_{\operatorname{aut}(\mathbb{D})} \omega \circ \phi(0)\left|\phi^{\prime}(0)\right|^{2} d \phi & =\int_{\mathbb{T} \times \mathbb{D}} \omega(\beta \lambda)\left(1-|\lambda|^{2}\right)^{2} \frac{d \sigma(\beta) d \Sigma(\lambda)}{\left(1-|\lambda|^{2}\right)^{2}} \\
& =\int_{\mathbb{T} \times \mathbb{D}} \omega(\beta \lambda) d \sigma(\beta) d \Sigma(\lambda)=\int_{\mathbb{T}} \omega(0) d \sigma(\beta)=1 .
\end{aligned}
$$

We want $\omega_{\Phi}$ to approximate $\omega$ in $L^{1}(\mathbb{D})$ norm, and to be real-analytic on $\mathbb{D}$. The first aim is reached by letting $\Phi$ have most of its mass concentrated near the unit element of the group aut $(\mathbb{D})$, which in the coordinates $\phi=R_{\beta} \circ \phi_{\lambda}$ corresponds to $\lambda=0$ and $\beta=-1$. That means that the parameter $N$ should be very large for the function $\Phi_{2}$ given by (4.2) to be concentrated near 0 in $\mathbb{D}$, and that the function $\Phi_{1}$ given by (4.3) should have most of its mass near the point -1 on the unit circle, which happens if the parameter $\varrho$ is close to 1 . It is helpful to know that the contribution of remote elements $\phi$ to the integral (4.5) defining $\omega_{\Phi}$ is small: here we can use the a priori bound in Lemma 2.2 on $\omega$ and the fact that the function $\Phi_{2}(\lambda)$ drops off quickly as $\lambda$ approaches $\mathbb{T}$. To deal with the second aim, we proceed as follows. For $\phi=R_{\beta} \circ \phi_{\lambda}$,

$$
\phi \circ \phi_{z}=R_{\beta \gamma} \circ \phi_{\phi_{z}(\lambda)}, \quad \text { with } \gamma=\frac{\phi_{\lambda}(z)}{\phi_{z}(\lambda)}=\frac{\lambda \bar{z}-1}{1-\bar{\lambda} z},
$$

so that

$$
\Phi\left(\phi \circ \phi_{z}\right)=\Phi_{1}(\beta \gamma) \Phi_{2}\left(\phi_{z}(\lambda)\right), \quad \phi=R_{\beta} \circ \phi_{\lambda}
$$

We shall need the following: the functions $\Phi\left(\phi \circ \phi_{z}\right)$, considered as functions of $z \in \mathbb{D}$, should extend holomorphically to a neighborhood $U$ of $\mathbb{D} \subset \mathbb{R}^{2}$ in $\mathbb{C}^{2}$, and be uniformly bounded there. We shall do this locally around an arbitrary point $z_{0} \in \mathbb{D}$. A calculation shows that

$$
\Phi_{2}\left(\phi_{z}(\lambda)\right)=(N-1) \frac{\left(1-|z|^{2}\right)^{N}\left(1-|\lambda|^{2}\right)^{N}}{|1-\bar{\lambda} z|^{2 N}} .
$$


Real-analytic functions on $\mathbb{D}$ are functions of the type $F(z, \bar{z})$, where $F$ is a holomorphic function of two variables in a neighborhood of the anti-diagonal $\{(z, \bar{z}): z \in \mathbb{D}\}$. A holomorphic extension of $\Phi_{2}\left(\phi_{z}(\lambda)\right)$ is supplied by the formula

$$
F\left(z, z^{*}\right)=(N-1) \frac{\left(1-z z^{*}\right)^{N}\left(1-|\lambda|^{2}\right)^{N}}{(1-\bar{\lambda} z)^{N}\left(1-\lambda z^{*}\right)^{N}},
$$

which is bounded uniformly in $\lambda \in \mathbb{D}$ provided that $z$ is close to $z_{0}$ and $z^{*}$ is close to $\bar{z}_{0}$. Let $\Phi_{1}$ denote not only the real-analytic function on $\mathbb{T}$ but also its bounded holomorphic extension to a neighborhood of $\mathbb{T}$; with $\Phi_{1}$ given by (4.3), the extension is

$$
\Phi_{1}(\beta)=\frac{1-\varrho^{2}}{(1+\varrho \beta)(1+\varrho / \beta)}, \quad \beta \in \mathbb{C} \backslash\left\{-\varrho,-\varrho^{-1}\right\} .
$$

A holomorphic extension of $G(z, \bar{z})=\Phi_{1}(\beta \gamma)$, where $\gamma$ is given by (4.6), is then given by

$$
G\left(z, z^{*}\right)=\Phi_{1}\left(\beta \frac{\lambda z^{*}-1}{1-\bar{\lambda} z}\right)
$$

which is also uniformly bounded in $\lambda \in \mathbb{D}$ provided that $z$ is close to $z_{0}$ and $z^{*}$ is close to $\bar{z}_{0}$.

It follows that with the above choice of $\Phi, \omega_{\Phi}$ approximates $\omega$ in the $L^{1}(\mathbb{D})$ norm, is reproducing, logarithmically subharmonic, and real-analytic on $\mathbb{D}$.

The effect of dilatation. We wish to approximate a given weight $\omega$ on $\mathbb{D}$, which is logarithmically subharmonic and reproducing, by a positive weight which is real-analytic on the closed disk $\overline{\mathbb{D}}$, and has the same properties. From the previous section we know that we can achieve real-analyticity in the interior $\mathbb{D}$. We can therefore assume from the start that $\omega$ is real-analytic and positive on $\mathbb{D}$.

For $r, 0<r<1$, let $\omega_{r}(z)=\omega(r z)$ be the associated dilatation of $\omega$. We shall see that $\omega_{r}$ is subrepresenting, that is, that for all positive bounded harmonic functions $h$ on $\mathbb{D}$, we have

$$
\int_{\mathbb{D}} h(z) \omega_{r}(z) d \Sigma(z) \leqq h(0)
$$

Let $P(z, \zeta)$ denote the Poisson kernel

$$
P(z, \zeta)=\frac{1-|z|^{2}}{|1-z \bar{\zeta}|^{2}}, \quad(z, \zeta) \in \mathbb{D} \times \mathbb{T},
$$

and consider, for $\lambda \in \mathbb{D}$, the function

$$
\int_{\mathbb{T}} P(\lambda, \alpha) \omega(\alpha z) d \sigma(\alpha), \quad z \in \mathbb{D}
$$


As a function of $\lambda$, this function is harmonic and equals $\omega(\lambda z)$ for $\lambda \in \mathbb{T}$. As the function $\omega(\lambda z)$ is subharmonic in the variable $\lambda$, it follows that

$$
\omega(\lambda z) \leqq \int_{\mathbb{T}} P(\lambda, \alpha) \omega(\alpha z) d \sigma(\alpha), \quad(z, \lambda) \in \mathbb{D} \times \mathbb{D}
$$

We specialize to $\lambda=r, 0<r<1$ :

$$
\omega_{r}(z) \leqq \int_{\mathbb{T}} P(r, \alpha) \omega(\alpha z) d \sigma(\alpha), \quad z \in \mathbb{D}
$$

By the reproducing property of $\omega$, we get for all positive bounded harmonic functions $h$ on $\mathbb{D}$ that

$$
\begin{aligned}
\int_{\mathbb{D}} h(z) \omega_{r}(z) d \Sigma(z) & \leqq \int_{\mathbb{T} \times \mathbb{D}} h(z) P(r, \alpha) \omega(\alpha z) d \Sigma(z) d \sigma(\alpha) \\
& =\int_{\mathbb{T}} P(r, \alpha) \int_{\mathbb{D}} h(z) \omega(\alpha z) d \Sigma(z) d \sigma(\alpha) \\
& =\int_{\mathbb{T}} P(r, \alpha) h(0) d \sigma(\alpha)=h(0),
\end{aligned}
$$

as asserted above.

Completing subrepresenting weights. We now complete the subrepresenting weight $\omega_{r}$ by adding a suitable small term which makes the sum representing. We consider first the harmonic function

$$
P^{*}\left[\omega_{r}\right](z)=\int_{\mathbb{D}} P(z, \zeta) \omega_{r}(\zeta) d \Sigma(\zeta), \quad z \in \mathbb{D}
$$

where we have extended the Poisson kernel to the interior:

$$
P(z, \zeta)=\frac{1-|z \zeta|^{2}}{|1-z \bar{\zeta}|^{2}}, \quad(z, \zeta) \in \mathbb{D} \times \mathbb{D} .
$$

The function $P^{*}\left[\omega_{r}\right]$ extends harmonically to a neighborhood of the closed unit disk. One way to see this is to realize that $\left.P^{*}\left[\omega_{r}\right]\right|_{\mathbb{T}}$ is the outward normal derivative of the function

$$
G\left[\omega_{r}\right](z)=\int_{\mathbb{D}} G(z, \zeta) \omega_{r}(\zeta) d \Sigma(\zeta), \quad z \in \mathbb{D}
$$

which solves the problem $\Delta G\left[\omega_{r}\right]=\omega_{r}$ with boundary data $\left.G\left[\omega_{r}\right]\right|_{\mathbb{T}}=0$. Here, $G(\cdot, \cdot)$ denotes the Green function for the laplacian $\Delta$ on $\mathbb{D}$. By a classical theorem of Painlevé, the real-analyticity of the data $\omega_{r}$ forces the real-analyticity of the solution $G\left[\omega_{r}\right]$, also on the boundary (see also [32]). The assertion that $P^{*}\left[\omega_{r}\right]$ is real-analytic and hence harmonic on $\overline{\mathbb{D}}$ is immediate. By the subrepresenting property (4.7) of $\omega_{r}, 0<P^{*}\left[\omega_{r}\right] \leqq 1$ throughout $\mathbb{D}$, and hence we have $0 \leqq P^{*}\left[\omega_{r}\right] \leqq 1$ also on $\mathbb{T}$. Let $\theta$ be a real parameter with $0<\theta<1$, and consider the function $H(z)=1-\theta P^{*}\left[\omega_{r}\right](z)$, which is harmonic, bounded above by 1 , and positive, in a neighborhood of $\overline{\mathbb{D}}$. Let $\varrho, 1<\varrho<+\infty$, be so close to 1 that $H$ is harmonic on the dilated disk $\varrho \mathbb{D}$. Then the function 


$$
F(z)=\int_{\mathbb{T}} \frac{\left(1-\varrho^{-2}\right)^{2}}{\left|1-\varrho^{-1} z \bar{\zeta}\right|^{4}} H(\varrho \zeta) d \sigma(\zeta), \quad z \in \varrho \mathbb{D},
$$

is real-analytic in $\varrho \mathbb{D}$ and $F$ is positive there. Moreover, $F$ is logarithmically subharmonic, and for $z \in \mathbb{D}$,

$$
\begin{aligned}
P^{*}[F](z) & =\int_{\mathbb{D}} P(z, \zeta) F(\zeta) d \Sigma(\zeta)=\int_{\mathbb{D}} P(z, \zeta) \int_{\mathbb{W}} \frac{\left(1-\varrho^{-2}\right)^{2}}{\left|1-\varrho^{-1} \zeta \bar{\xi}\right|^{4}} H(\varrho \xi) d \sigma(\xi) d \Sigma(\zeta) \\
& =\int_{\mathbb{T}} \int_{\mathbb{D}} P(z, \zeta) \frac{\left(1-\varrho^{-2}\right)^{2}}{\left|1-\varrho^{-1} \zeta \bar{\xi}\right|^{4}} d \Sigma(\zeta) H(\varrho \xi) d \sigma(\xi)=\int_{\mathbb{T}} P\left(\varrho^{-1} z, \xi\right) H(\varrho \xi) d \sigma(\xi)=H(z) .
\end{aligned}
$$

It follows that the weight

$$
\tilde{\omega}(z)=\theta \omega_{r}(z)+F(z), \quad z \in \mathbb{D},
$$

is logarithmically subharmonic, strictly positive, and real-analytic on some neighborhood of $\overline{\mathbb{D}}$. It also has $P^{*}[\tilde{\omega}]=1$, which is another way of expressing that $\tilde{\omega}$ is reproducing:

$$
\int_{\mathbb{D}} h(z) \tilde{\omega}(z) d \Sigma(z)=h(0),
$$

first for all bounded and positive harmonic functions $h$, then in a second step, for all bounded harmonic functions $h$ on $\mathbb{D}$.

We now look at the $L^{1}(\mathbb{D})$ norm proximity to the original weight $\omega$. If the parameter $r, 0<r<1$, is close to 1 , the dilate $\omega_{r}$ is close to $\omega$. Also, if $\theta$ is close to 1 , the function $\theta \omega_{r}$ still approximates $\omega$ well. But this means that $\theta P^{*}\left[\omega_{r}\right](0)$ is close to 1 , and as the $L^{1}(\mathbb{D})$ norm of $F$ equals the difference $1-P^{*}\left[\omega_{r}\right](0)$, the modified weight $\tilde{\omega}$ approximates $\omega$ well in $L^{1}(\mathbb{D})$.

\section{The approximation of Green functions}

Let $\omega$ be logarithmically subharmonic on the unit disk $\mathbb{D}$ and reproducing (for the origin). We wish to show that the biharmonic Green function $\Gamma_{\omega}$ for the fourth order elliptic operator $\Delta \omega^{-1} \Delta$ with vanishing Dirichlet data is positive. By Theorem 4.1, $\omega$ can be approximated in the $L^{1}(\mathbb{D})$ norm by a weight $\tilde{\omega}$ which in addition to being logarithmically subharmonic and reproducing is real-analytic and strictly positive on $\overline{\mathbb{D}}$. We need to show that the corresponding Green functions $\Gamma_{\omega}$ and $\Gamma_{\tilde{\omega}}$ are appropriately close.

We shall need the following basic estimate of the kernel $K_{\omega}$, obtained by Hedenmalm in [22].

Theorem 5.1. Let $\omega$ be a logarithmically subharmonic weight which is reproducing for the origin. Then

$$
\left|K_{\omega}(z, \zeta)\right| \leqq \frac{2}{|1-z \bar{\zeta}|^{2}}, \quad(z, \zeta) \in \mathbb{D} \times \mathbb{D}
$$


Theorem 5.2. Let $\omega$ and $\omega_{n}$, for $n=1,2,3, \ldots$, be logarithmically subharmonic weights which reproduce for the origin. If $\omega_{n} \rightarrow \omega$ in the norm of $L^{1}(\mathbb{D})$ as $n \rightarrow+\infty$, then $\Gamma_{\omega_{n}}(z, \zeta) \rightarrow \Gamma_{\omega}(z, \zeta)$ pointwise in $\mathbb{D} \times \mathbb{D}$ as $n \rightarrow+\infty$.

Proof. Let $v$ denote a weight of the same general type as $\omega$ and $\omega_{n}$, and recall that by the reproducing property of $v$, we have the following identity of reproducing kernel functions (see Proposition 2.3):

$$
Q_{v}(z, \zeta)=2 \operatorname{Re} K_{v}(z, \zeta)-1, \quad(z, \zeta) \in \mathbb{D} \times \mathbb{D} .
$$

As in Definition 3.1, let $H_{v}(\cdot, \zeta)$ be the function

$$
H_{v}(z, \zeta)=-\int_{\mathbb{D}} Q_{v}(z, \eta) G(\eta, \zeta) v(\eta) d \Sigma(\eta), \quad(z, \zeta) \in \mathbb{D} \times \mathbb{D}
$$

so that

$$
\text { (5.2) } \Gamma_{v}(z, \zeta)=\int_{\mathbb{D}} G(z, \xi)\left[G(\xi, \zeta)+H_{v}(\xi, \zeta)\right] v(\xi) d \Sigma(\xi), \quad(z, \zeta) \in \mathbb{D} \times \mathbb{D}
$$

By Theorem 5.1 and the relationship (5.1), we have the estimate

$$
\left|Q_{v}(z, \zeta)\right| \leqq 1+\frac{4}{|1-z \bar{\zeta}|^{2}}, \quad(z, \zeta) \in \mathbb{D} \times \mathbb{D}
$$

We shall use this to estimate the size of the kernel $H_{v}$. We observe that by the reproducing property of $v$,

$$
\int_{\mathbb{D}} \frac{1-|z \eta|^{2}}{|1-z \bar{\eta}|^{2}} v(\eta) d \Sigma(\eta)=1, \quad z \in \mathbb{D},
$$

and that by Fatou's lemma, the integral on the left hand side is bounded by 1 for $z \in \mathbb{T}$. For $\zeta$ confined to a compact subset $X$ of $\mathbb{D}$, the Green function $G(\eta, \zeta)$ is comparable to $-\left(1-|\eta|^{2}\right)$ near the boundary, which allows us to use estimate (5.3) in conjunction with (5.4) to obtain the uniform estimate

$$
\left|H_{v}(z, \zeta)\right| \leqq C(X), \quad z \in \mathbb{D}, \zeta \in X
$$

for some positive constant $C(X)$ depending on $X$, universal for all the weights $v$. We now show that $H_{\omega_{n}}(\cdot, \zeta) \rightarrow H_{\omega}(\cdot, \zeta)$ in an appropriate norm. For fixed $\zeta \in \mathbb{D}$, the function $F_{v}(\cdot, \zeta)$, defined by

$$
F_{v}(z, \zeta)=G(z, \zeta)+H_{v}(z, \zeta)
$$

is perpendicular to the bounded harmonic functions in the scalar product of $L^{2}(\mathbb{D}, v)$. Using this fact and the estimate (5.5), we arrive at the identities

$$
\int_{\mathbb{D}}\left|H_{\omega}(z, \zeta)-H_{\omega_{n}}(z, \zeta)\right|^{2} \omega(z) d \Sigma(z)=\int_{\mathbb{D}}\left(\left|F_{\omega_{n}}(z, \zeta)\right|^{2}-\left|F_{\omega}(z, \zeta)\right|^{2}\right) \omega(z) d \Sigma(z),
$$


and

$$
\int_{\mathbb{D}}\left|H_{\omega}(z, \zeta)-H_{\omega_{n}}(z, \zeta)\right|^{2} \omega_{n}(z) d \Sigma(z)=\int_{\mathbb{D}}\left(\left|F_{\omega}(z, \zeta)\right|^{2}-\left|F_{\omega_{n}}(z, \zeta)\right|^{2}\right) \omega_{n}(z) d \Sigma(z)
$$

We add these together, and obtain

$$
\begin{aligned}
\int_{\mathbb{D}}\left|H_{\omega}(z, \zeta)-H_{\omega_{n}}(z, \zeta)\right|^{2}\left(\omega(z)+\omega_{n}(z)\right) d \Sigma(z) \\
\quad=\int_{\mathbb{D}}\left(\left|F_{\omega}(z, \zeta)\right|^{2}-\left|F_{\omega_{n}}(z, \zeta)\right|^{2}\right)\left(\omega_{n}(z)-\omega(z)\right) d \Sigma(z) .
\end{aligned}
$$

By the uniform estimate (5.5), the growth estimate of reproducing logarithmically subharmonic weights of Lemma 2.2, and the $L^{1}(\mathbb{D})$ convergence $\omega_{n} \rightarrow \omega$, it follows that uniformly in $\zeta \in X$,

$$
\int_{\mathbb{D}}\left|H_{\omega}(z, \zeta)-H_{\omega_{n}}(z, \zeta)\right|^{2} \omega(z) d \Sigma(z) \rightarrow 0 \quad \text { as } n \rightarrow+\infty
$$

or in other words, $H_{\omega_{n}}(\cdot, \zeta) \rightarrow H_{\omega}(\cdot, \zeta)$ in the norm of $L^{2}(\mathbb{D}, \omega)$. By $(5.2)$,

$$
\begin{aligned}
\Gamma_{\omega}(z, \zeta)-\Gamma_{\omega_{n}}(z, \zeta)= & \int_{\mathbb{D}} G(z, \xi)\left[H_{\omega}(\xi, \zeta)-H_{\omega_{n}}(\xi, \zeta)\right] \omega(\xi) d \Sigma(\xi) \\
& +\int_{\mathbb{D}} G(z, \xi)\left[G(\xi, \zeta)+H_{\omega_{n}}(\xi, \zeta)\right]\left(\omega(\xi)-\omega_{n}(\xi)\right) d \Sigma(\xi),(z, \zeta) \in \mathbb{D} \times \mathbb{D},
\end{aligned}
$$

so that the desired result follows from (5.6), (5.5), the growth estimate of Lemma 2.2, and the $L^{1}(\mathbb{D})$ convergence $\omega_{n} \rightarrow \omega$.

\section{Bergman kernels: structural properties}

The general theory of reproducing kernels. In the general theory of reproducing kernel functions (see Saitoh's book [37]), introduced and studied by Mercer, Moore, Aronszajn, Kreìn, and Schwartz, a complex-valued function $K$ of two variables, say $(x, y)$, defined on some product set $E \times E$, is said to be a reproducing kernel if for any finite subset $\left\{x_{1}, x_{2}, \ldots, x_{N}\right\}$ of $E$, we have that the matrix

$$
\left\{K\left(x_{j}, x_{k}\right)\right\}_{j, k=1}^{N}
$$

is positive definite, in other words, that

$$
0 \leqq \sum_{j, k=1}^{N} K\left(x_{j}, x_{k}\right) w_{j} \bar{w}_{k}
$$

holds for all sequences $\left\{w_{j}\right\}_{j=1}^{N} \in \mathbb{C}^{N}$. In particular, such a kernel has $0 \leqq K(x, x)$, $K(x, y)=\bar{K}(y, x)$, and

$$
|K(x, y)| \leqq K(x, x)^{\frac{1}{2}} K(y, y)^{\frac{1}{2}}
$$


for all $x$ and $y$ in $E$. The above definition does not refer to any Hilbert space of functions with bounded point evaluations, which was the way we defined the reproducing kernels for the Bergman spaces back in Section 2. It turns out that if we have a Hilbert space of functions with bounded point evaluations, then its reproducing kernel function has the above positive definiteness property, and that if on the other hand, we have a reproducing kernel function as above, there exists a unique Hilbert space for which it reproduces the point evaluation functionals, by a theorem ascribed to Moore and Aronszajn [37]. For instance, if we take a subspace $\subseteq$ with the properties assumed in Section 2, and write down an orthonormal basis $\left\{\varphi_{j}\right\}_{j=1}^{\infty}$ for $\mathfrak{S}^{2}(\Omega, \omega)$, then by the formula for the kernel in terms of the basis,

$$
0 \leqq \sum_{l=1}^{\infty}\left|\sum_{j=1}^{N} \varphi_{l}\left(z_{j}\right) w_{j}\right|^{2}=\sum_{l=1}^{\infty} \sum_{j, k=1}^{N} \varphi_{l}\left(z_{j}\right) \bar{\varphi}_{l}\left(z_{k}\right) w_{j} \bar{w}_{k}=\sum_{j, k=1}^{N} K^{\Im}\left(z_{j}, z_{k}\right) w_{j} \bar{w}_{k}
$$

The following general result is of some interest; it is known, but we do not have a reference. An infinite matrix $\{A(j, k)\}_{j, k=0}^{\infty}$ is said to be positive definite if each finite submatrix $\{A(j, k)\}_{j, k=0}^{N-1}$ is positive definite, that is, for any finite sequence of points $\left\{\alpha_{j}\right\}_{j=0}^{N-1} \in \mathbb{C}^{N}$, we have that

$$
0 \leqq \sum_{j, k=1}^{N-1} A(j, k) \alpha_{j} \bar{\alpha}_{k}
$$

Proposition 6.1. Let $K$ be a function with a convergent power series expansion on the bidisk $\mathbb{D}^{2}$,

$$
K(z, \zeta)=\sum_{j, k=0}^{\infty} \hat{K}(j, k) z^{j \bar{\zeta}^{k}}, \quad(z, \zeta) \in \mathbb{D} \times \mathbb{D}
$$

Then $K$ is a reproducing kernel on $\mathbb{D} \times \mathbb{D}$ if and only if the infinite matrix $\{\hat{K}(j, k)\}_{j, k=0}^{\infty}$ is positive definite.

Proof. Let $\left\{w_{j}\right\}_{j=1}^{N} \in \mathbb{C}^{N}$ be arbitrary, and put

$$
\alpha_{m}=\sum_{j=1}^{N} \omega_{j} z_{j}^{m}
$$

where $\left\{z_{j}\right\}_{j=1}^{N}$ is a given sequence of points in $\mathbb{D}$. Then a change of the order of summation shows that

$$
\sum_{j, k=1}^{N} K\left(z_{j}, z_{k}\right) w_{j} \bar{w}_{k}=\sum_{m, n=0}^{\infty} \hat{K}(m, n) \sum_{j, k=1}^{N} w_{j} \bar{w}_{k} z_{j}^{m} \bar{z}_{k}^{n}=\sum_{m, n=0}^{\infty} \hat{K}(m, n) \alpha_{j} \bar{\alpha}_{k} .
$$

One implication is immediate: if $\{\hat{K}(m, n)\}_{m, n=0}^{\infty}$ is positive definite, then $K$ is a reproducing kernel. We turn to the reverse implication. Given a sequence $\left\{\alpha_{m}\right\}_{m=1}^{N} \in \mathbb{C}^{N}$, we would like to find points $z_{1}, \ldots, z_{N}$ in $\mathbb{D}$ and a sequence $\left\{w_{j}\right\}_{j=1}^{N} \in \mathbb{C}^{N}$ such that (6.2) holds for $m=0,1,2, \ldots, N$, because then the reverse implication also follows from the above 
identity. This can easily be accomplished by choosing the points equidistantly on a concentric circle of radius $r, 0<r<1$,

$$
z_{j}=r e^{2 \pi i j / N}, \quad j=1,2, \ldots, N
$$

because then we can use Fourier analysis on finite commutative groups to find expressions for $w_{j}$ in terms of the $\alpha_{m}$ 's so as to have the desired relation between these two finite sequences.

Reproducing kernels for weighted Bergman spaces. In the rest of the section, we shall be concerned with weights $\omega: \mathbb{D} \rightarrow[0,+\infty[$ which are area-summable on $\mathbb{D}$ and meet the following two conditions:

- $\omega$ is logarithmically subharmonic on $\mathbb{D}$, and

- $\omega$ is reproducing for the origin.

We shall study the reproducing kernel $K_{\omega}$ for the Hilbert space $P^{2}(\mathbb{D}, \omega)$ in detail under these assumptions.

The following structure result is a well known consequence of the fact that the shift operator $S f(z)=z f(z)$ is contractive on $P^{2}(\mathbb{D}, \omega)$ (see Saitoh [37], p. 135).

Theorem 6.2. The function $J_{\omega}(z, \zeta)=(1-z \bar{\zeta}) K_{\omega}(z, \zeta)$ is the reproducing kernel for a Hilbert space of holomorphic functions on $\mathbb{D}$.

The similar-looking structure result below is key to our further investigations.

Theorem 6.3. The function $L_{\omega}$ defined by the equality

$$
K_{\omega}(z, \zeta)=\frac{1-z \bar{\zeta} L_{\omega}(z, \zeta)}{(1-z \bar{\zeta})^{2}}
$$

is the reproducing kernel for a Hilbert space of holomorphic functions on $\mathbb{D}$.

We postpone the proof a little. First, we need the following important property of the shift operator $S, S f(z)=z f(z)$, acting on $P^{2}(\mathbb{D}, \omega)$.

Proposition 6.4. For any two functions $f, g \in P^{2}(\mathbb{D}, \omega)$, we have the inequality

$$
\|S f+g\|_{\omega}^{2} \leqq 2\left(\|f\|_{\omega}^{2}+\|S g\|_{\omega}^{2}\right)
$$

Proof. It is enough to obtain the inequality when $f$ and $g$ are polynomials. Let us first assume the weight $\omega$ is $C^{\infty}$-smooth up to the boundary. For any $\lambda \in \mathbb{C} \backslash\{0\}$, we have that

$$
\begin{aligned}
0 & \leqq \Delta_{z}\left(\left|g(z)-\lambda^{-1} z^{3} f(z)\right|^{2} \omega(z)\right) \\
& =\Delta_{z}\left(|g(z)|^{2} \omega(z)\right)-2 \operatorname{Re}\left(\bar{\lambda}^{-1} \Delta_{z}\left(g(z) \bar{z}^{3} \bar{f}(z) \omega(z)\right)\right)+|\lambda|^{-2} \Delta_{z}\left(\left|z^{3} f(z)\right|^{2} \omega(z)\right),
\end{aligned}
$$


for $z \in \mathbb{D}$, where the first inequality holds because the product of a logarithmically subharmonic function and the modulus-squared of a holomorphic function is again logarithmically subharmonic, and in particular, subharmonic. Substituting $\lambda=z^{2}$ in (6.3), we obtain

$$
\begin{aligned}
0 \leqq & \Delta_{z}\left(|g(z)|^{2} \omega(z)\right)-2 \operatorname{Re}\left(\bar{z}^{-2} \Delta_{z}\left(g(z) \bar{z}^{3} \bar{f}(z) \omega(z)\right)\right) \\
& +|z|^{-4} \Delta_{z}\left(\left|z^{3} f(z)\right|^{2} \omega(z)\right) .
\end{aligned}
$$

We note that none of the three terms on the right hand side has the slightest singularity at the origin, even though it may seem so to the inexperienced eye. By Green's formula,

$$
\int_{\mathbb{D}}\left(1-|z|^{2}\right)^{2} \Delta_{z}\left(|g(z)|^{2} \omega(z)\right) d \Sigma(z)=\int_{\mathbb{D}}\left(4|z|^{2}-2\right)|g(z)|^{2} \omega(z) d \Sigma(z) .
$$

A slightly more sophisticated exercise involving Green's formula shows that if $\mathbb{D}(0, \varepsilon)$ stands for a small circular disk about the origin of radius $\varepsilon$, then

$$
\begin{aligned}
\int_{\mathbb{D} \backslash \mathbb{D}(0, \varepsilon)} & \left(1-|z|^{2}\right)^{2}\left(\bar{z}^{-2} \Delta_{z}\left(g(z) \bar{z}^{3} \bar{f}(z) \omega(z)\right)\right) d \Sigma(z) \\
= & \int_{\mathbb{D} \backslash \mathbb{D}(0, \varepsilon)} \Delta_{z}\left(\bar{z}^{-2}\left(1-|z|^{2}\right)^{2}\right)\left(g(z) \bar{z}^{3} \bar{f}(z) \omega(z)\right) d \Sigma(z) \\
& +2 \int_{\partial \mathbb{D}(0, \varepsilon)}\left(\left(1-|z|^{2}\right)^{2} \bar{z}^{-2} \partial_{n(z)}\left(g(z) \bar{z}^{3} \bar{f}(z) \omega(z)\right)\right. \\
& \left.-\partial_{n(z)}\left(\left(1-|z|^{2}\right)^{2} \bar{z}^{-2}\right) g(z) \bar{z}^{3} \bar{f}(z) \omega(z)\right) d \sigma(z) \\
= & \int_{\mathbb{D} \backslash \mathbb{D}(0, \varepsilon)} \bar{z} g(z) \bar{f}(z) \omega(z) d \Sigma(z)+O(\varepsilon),
\end{aligned}
$$

as $\varepsilon \rightarrow 0$, where the normal derivative is taken inward with respect to the disk $\mathbb{D}(0, \varepsilon)$.

We apply Green's formula a third time, and obtain

$$
\begin{aligned}
\int_{\mathbb{D} \backslash \mathbb{D}(0, \varepsilon)} & \left(1-|z|^{2}\right)^{2}\left(|z|^{-4} \Delta_{z}\left(\left|z^{3} f(z)\right|^{2} \omega(z)\right)\right) d \Sigma(z) \\
= & \int_{\mathbb{D} \backslash \mathbb{D}(0, \varepsilon)} \Delta_{z}\left(|z|^{-4}\left(1-|z|^{2}\right)^{2}\right)\left|z^{3} f(z)\right|^{2} \omega(z) d \Sigma(z) \\
& +2 \int_{\partial \mathbb{D}(0, \varepsilon)}\left(\left(1-|z|^{2}\right)^{2}|z|^{-4} \partial_{n(z)}\left(\left|z^{3} f(z)\right|^{2} \omega(z)\right)\right. \\
= & \left.\quad-\left|z^{3} f(z)\right|^{2} \omega(z) \partial_{n(z)}\left(\left(1-|z|^{2}\right)^{2}|z|^{-4}\right)\right) d \sigma(z) \\
& \int_{\mathbb{D} \backslash \mathbb{D}(0, \varepsilon)}\left(4-2|z|^{2}\right)|f(z)|^{2} \omega(z) d \Sigma(z)+O(\varepsilon),
\end{aligned}
$$

as $\varepsilon \rightarrow 0$. Putting the terms (6.5)-(6.7) together, using (6.4), we arrive in the limit $\varepsilon \rightarrow 0$ at 


$$
\begin{aligned}
0 \leqq & \int_{\mathbb{D}}\left(4|z|^{2}-2\right)|g(z)|^{2} \omega(z) d \Sigma(z) \\
& \quad-4 \operatorname{Re} \int_{\mathbb{D}} \bar{z} g(z) \bar{f}(z) \omega(z) d \Sigma(z)+\int_{\mathbb{D}}\left(4-2|z|^{2}\right)|f(z)|^{2} \omega(z) d \Sigma(z),
\end{aligned}
$$

which expresses in expanded form the inequality we are looking for. We now turn to the explanation of why we can assume $\omega$ to be $C^{\infty}$-smooth. From the previous section, we know that we can approximate $\omega$ in the $L^{1}(\mathbb{D})$-norm with weights of the same type but with a much higher degree of smoothness $\left(C^{\omega}\right.$ on $\overline{\mathbb{D}}$, in fact). And since we only need to check the above inequality (6.8) for fixed polynomials $f, g$ at a time, the assertion is immediate.

In addition to the forward shift $S$, we shall need the backward shift $T$, as defined by

$$
T f(z)=\frac{f(z)-f(0)}{z}, \quad z \in \mathbb{D}
$$

which we think of as acting on $P^{2}(\mathbb{D}, \omega)$. The composed operator $T S$ is the identity, and $S T$ is given by $\operatorname{STf}(z)=f(z)-f(0)$. The forward shift $S$ is a contraction on $P^{2}(\mathbb{D}, \omega)$, and so is $S T$, because of the reproducing property of the weight $\omega$, which leads to the norm identity

$$
\|f\|_{\omega}^{2}=\|f-f(0)\|_{\omega}^{2}+|f(0)|^{2}, \quad f \in P^{2}(\mathbb{D}, \omega) .
$$

The variant of Proposition 6.4 which we shall actually use is the following.

Corollary 6.5. For any two functions $f, g \in P^{2}(\mathbb{D}, \omega)$, we have the inequality

$$
\|S f+T g\|_{\omega}^{2} \leqq 2\left(\|f\|_{\omega}^{2}+\|g\|_{\omega}^{2}\right) .
$$

We are now ready to prove Theorem 6.3.

Proof. Solving for $L_{\omega}$, we find that

$$
\begin{aligned}
L_{\omega}(z, \zeta) & =\frac{1}{z \bar{\zeta}}\left(1-(1-z \bar{\zeta})^{2} K_{\omega}(z, \zeta)\right) \\
& =\frac{1-K_{\omega}(z, \zeta)}{z \bar{\zeta}}+2 K_{\omega}(z, \zeta)-z \bar{\zeta} K_{\omega}(z, \zeta)
\end{aligned}
$$

By the reproducing property of the weight $\omega$, we have that

$$
K_{\omega}(z, 0)=K_{\omega}(0, \zeta)=1, \quad(z, \zeta) \in \mathbb{D} \times \mathbb{D},
$$

so that by some well-known division properties of holomorphic function on the bidisk $\mathbb{D}^{2}$, the function $L_{\omega}(z, \bar{\zeta})$ is holomorphic on $\mathbb{D}^{2}$. As a consequence of Theorem 5.1, the kernel

$$
z \bar{\zeta} L_{\omega}(z, \zeta)=1-(1-z \bar{\zeta})^{2} K_{\omega}(z, \zeta), \quad(z, \zeta) \in \mathbb{D} \times \mathbb{D}
$$


is bounded in modulus by 3 , so that by the maximum principle for holomorphic functions of two complex variables,

$$
\left|L_{\omega}(z, \zeta)\right| \leqq 3, \quad(z, \zeta) \in \mathbb{D} \times \mathbb{D}
$$

We shall see later that the bound 3 may be replaced by 1 , which is best possible.

We wish to prove that $L_{\omega}$ is a reproducing kernel. In other words, we should show that for any finite subset $\left\{z_{1}, z_{2}, \ldots, z_{N}\right\}$ of $\mathbb{D}$, it is the case that

$$
0 \leqq \sum_{j, k=1}^{N} L_{\omega}\left(z_{j}, z_{k}\right) w_{j} \bar{w}_{k}
$$

holds for all sequences $\left\{w_{j}\right\}_{j=1}^{N} \in \mathbb{C}^{N}$. By exploiting the reproducing property of the kernel $K_{\omega}$, we have that

$$
L_{\omega}\left(z_{j}, z_{k}\right)=\int_{\mathbb{D} \times \mathbb{D}} L_{\omega}(z, \zeta) K_{\omega}\left(z_{j}, z\right) K_{\omega}\left(\zeta, z_{k}\right) \omega(z) \omega(\zeta) d \Sigma(z) d \Sigma(\zeta)
$$

where the integral is absolutely convergent because of estimate (6.11) and the bound on $K_{\omega}$ from Theorem 5.1. If we let $f$ be the $H^{\infty}(\mathbb{D})$ function- $H^{\infty}(\mathbb{D})$ is the algebra of bounded holomorphic functions on $\mathbb{D}$ - given by the formula

$$
f(z)=\sum_{j=1}^{N} \bar{\omega}_{j} K_{\omega}\left(z, z_{j}\right), \quad z \in \mathbb{D}
$$

we see that $(6.12)$ is equivalent to having

$$
0 \leqq \int_{\mathbb{D} \times \mathbb{D}} L_{\omega}(z, \zeta) \bar{f}(z) f(\zeta) \omega(z) \omega(\zeta) d \Sigma(z) d \Sigma(\zeta)
$$

We shall obtain $(6.13)$ for all $f \in H^{\infty}(\mathbb{D})$. The forward and backward shift operators $S$ and $T$, acting on $P^{2}(\mathbb{D}, \omega)$, have adjoints $S_{\omega}^{*}$ and $T_{\omega}^{*}$, where the subscript indicates that the adjoint is taken with respect to the inner product of $P^{2}(\mathbb{D}, \omega)$ :

$$
\langle S f, g\rangle_{\omega}=\left\langle f, S_{\omega}^{*} g\right\rangle_{\omega}, \quad\langle T f, g\rangle_{\omega}=\left\langle f, T_{\omega}^{*} g\right\rangle_{\omega}, \quad \text { for } f, g \in P^{2}(\mathbb{D}, \omega)
$$

We have from (6.9) and (6.10) that for $f \in H^{\infty}(\mathbb{D})$,

$$
\begin{aligned}
\int_{\mathbb{D}} L_{\omega}(z, \zeta) f(\zeta) \omega(\zeta) d \Sigma(\zeta) \\
\quad=-\frac{1}{z}\left\langle f, T K_{\omega}(\cdot, z)\right\rangle_{\omega}+2\left\langle f, K_{\omega}(\cdot, z)\right\rangle_{\omega}-z\left\langle f, S K_{\omega}(\cdot, z)\right\rangle_{\omega} \\
\quad=-\frac{1}{z}\left\langle T_{\omega}^{*} f, K_{\omega}(\cdot, z)\right\rangle_{\omega}+2\left\langle f, K_{\omega}(\cdot, z)\right\rangle_{\omega}-z\left\langle S_{\omega}^{*} f, K_{\omega}(\cdot, z)\right\rangle_{\omega} \\
\quad=-\frac{T_{\omega}^{*} f(z)}{z}+2 f(z)-z S_{\omega}^{*} f(z), \quad z \in \mathbb{D}
\end{aligned}
$$


so that since $T_{\omega}^{*} f(0)=0$ - due to the reproducing property of $\omega$-we can condense the above to

$$
\int_{\mathbb{D}} L_{\omega}(z, \zeta) f(\zeta) \omega(\zeta) d \Sigma(\zeta)=-T T_{\omega}^{*} f(z)+2 f(z)-S S_{\omega}^{*} f(z), \quad z \in \mathbb{D}
$$

Integrating also with respect to the $z$ variable, we arrive at

$$
\begin{aligned}
& \int_{\mathbb{D} \times \mathbb{D}} L_{\omega}(z, \zeta) \bar{f}(z) f(\zeta) \omega(z) \omega(\zeta) d \Sigma(z) d \Sigma(\zeta) \\
& \quad=-\left\langle T T_{\omega}^{*} f, f\right\rangle_{\omega}+2\langle f, f\rangle_{\omega}-\left\langle S S_{\omega}^{*} f, f\right\rangle_{\omega}=-\left\|T_{\omega}^{*} f\right\|_{\omega}^{2}+2\|f\|_{\omega}^{2}-\left\|S_{\omega}^{*} f\right\|_{\omega}^{2}
\end{aligned}
$$

which shows that what we in fact need to know is that

$$
0 \leqq 2-T T_{\omega}^{*}-S S_{\omega}^{*}
$$

where the inequality is interpreted in the sense of operator theory (that is: $0 \leqq A$ means that $0 \leqq\langle A x, x\rangle$ holds for all space elements $x)$. Let $P^{2}(\mathbb{D}, \omega) \oplus P^{2}(\mathbb{D}, \omega)$ be the orthogonal sum of the two spaces, with elements $(f, g), f, g \in P^{2}(\mathbb{D}, \omega)$, and the inner product

$$
\left\langle\left(f_{1}, g_{1}\right),\left(f_{2}, g_{2}\right)\right\rangle_{\omega, \omega}=\left\langle f_{1}, f_{2}\right\rangle_{\omega}+\left\langle g_{1}, g_{2}\right\rangle_{\omega}
$$

We consider the operator $R: P^{2}(\mathbb{D}, \omega) \oplus P^{2}(\mathbb{D}, \omega) \rightarrow P^{2}(\mathbb{D}, \omega)$ given by

$$
R(f, g)=2^{-\frac{1}{2}}(S f+T g),
$$

and note that if $R_{\omega}^{*}: P^{2}(\mathbb{D}, \omega) \rightarrow P^{2}(\mathbb{D}, \omega) \oplus P^{2}(\mathbb{D}, \omega)$ is the adjoint defined by

$$
\langle R(f, g), h\rangle_{\omega}=\left\langle(f, g), R_{\omega}^{*} h\right\rangle_{\omega, \omega}, \quad f, g, h \in P^{2}(\mathbb{D}, \omega),
$$

then we have $R_{\omega}^{*}(h)=2^{-\frac{1}{2}}\left(S_{\omega}^{*} h, T_{\omega}^{*} h\right)$. It immediately follows that

$$
R R_{\omega}^{*} f=\frac{1}{2}\left(S S_{\omega}^{*} f+T T_{\omega}^{*} f\right), \quad f \in P^{2}(\mathbb{D}, \omega),
$$

so that the assertion (6.14) can be written as $R R_{\omega}^{*} \leqq 1$. This, however, is fulfilled precisely when $R_{\omega}^{*}$ is a contraction. By Corollary 6.5, the operator $R$ is a contraction, which implies that $R_{\omega}^{*}$ is a contraction as well. The proof is complete.

Corollary 6.6. Let the kernel $L_{\omega}$ be as in Theorem 6.3. Then

$$
\left|L_{\omega}(z, \zeta)\right|<1, \quad(z, \zeta) \in \mathbb{D}^{2}
$$

Proof. The identity

$$
K_{\omega}(z, z)=\frac{1-|z|^{2} L_{\omega}(z, z)}{\left(1-|z|^{2}\right)^{2}}, \quad z \in \mathbb{D},
$$


together with the observations that $0 \leqq K_{\omega}(z, z)$ and $0 \leqq L_{\omega}(z, z)$ shows that

$$
0 \leqq L_{\omega}(z, z) \leqq 1
$$

because the function $L_{\omega}(z, z)$ is subharmonic on $\mathbb{D}$. In fact, unless $L_{\omega}(z, z)$ equals the constant 1 identically on $\mathbb{D}$, we have a strict inequality: $L_{\omega}(z, z)<1$. And if $L_{\omega}(z, z) \equiv 1$, then $L_{\omega}(z, \zeta) \equiv 1$ too, because a kernel function is determined by its values along the diagonal. But if $L_{\omega}(z, \zeta) \equiv 1$, then the kernel $K_{\omega}$ must be the Hardy kernel (associated with the space $H^{2}(\mathbb{D})$ ), which cannot be, because $H^{2}(\mathbb{D})$ is not of the type $P^{2}(\mathbb{D}, \omega)$. We conclude that $\left|L_{\omega}(z, \zeta)\right|<1$, because after all, $L_{\omega}(z, z)$ is the norm-squared of the point evaluation functional at $z \in \mathbb{D}$ in the Hilbert space of holomorphic functions that can be associated with $L_{\omega}$.

We have achieved an improvement on the estimate of Theorem 5.1.

Corollary 6.7. The reproducing kernel $K_{\omega}$ can be estimated as follows:

$$
\left|K_{\omega}(z, \zeta)\right| \leqq \frac{1+|z \zeta|}{|1-z \bar{\zeta}|^{2}}, \quad(z, \zeta) \in \mathbb{D}^{2}
$$

Proof. This is immediate from Corollary 6.6.

The kernel $L_{\omega}$ has the following boundary behavior.

Theorem 6.8. Suppose our weight $\omega$ is continuous on $\overline{\mathbb{D}}$, in which case $1 \leqq\left.\omega\right|_{\mathbb{T}}$. Then the diagonal function $L_{\omega}(z, z)$ has a continuous extension to $\overline{\mathbb{D}}$, and the boundary values are

$$
L_{\omega}(z, z)=1-\frac{1}{\omega(z)}, \quad z \in \mathbb{T} .
$$

Proof. For $\lambda \in \mathbb{D}$, let $F_{\lambda}$ be the function

$$
F_{\lambda}(z)=\frac{1-|\lambda|^{2}}{(1-\bar{\lambda} z)^{2}}, \quad z \in \mathbb{D}
$$

which has norm 1 in $P^{2}(\mathbb{D})$. We fix a point $\zeta \in \mathbb{T}$. As $\lambda$ approaches $\zeta$ from the interior, $F_{\lambda}$ tends to 0 uniformly off every fixed neighborhood of the point $\zeta$, and consequently, the measure $\left|F_{\lambda}\right|^{2} d \Sigma$ tends to the unit point mass at $\zeta$. We apply this observation to integration against the weight $\omega$, and obtain

$$
\int_{\mathbb{D}}\left|F_{\lambda}(z)\right|^{2} \omega(z) d \Sigma(z) \rightarrow \omega(\zeta) \quad \text { as } \lambda \rightarrow \zeta
$$

On the other hand, we have the estimate

$$
\frac{1}{\left(1-|\lambda|^{2}\right)^{2}}=\left|F_{\lambda}(\lambda)\right|^{2} \leqq K_{\omega}(\lambda, \lambda) \int_{\mathbb{D}}\left|F_{\lambda}(z)\right|^{2} \omega(z) d \Sigma(z),
$$


so that

$$
\frac{1}{\omega(\zeta)} \leqq \liminf _{\lambda \rightarrow \zeta}\left(1-|\lambda|^{2}\right)^{2} K_{\omega}(\lambda, \lambda)=\liminf _{\lambda \rightarrow \zeta}\left(1-|\lambda|^{2} L_{\omega}(\lambda, \lambda)\right)=1-\limsup _{\lambda \rightarrow \zeta} L_{\omega}(\lambda, \lambda)
$$

which leads to half of the desired assertion,

$$
\limsup _{\lambda \rightarrow \zeta} L_{\omega}(\lambda, \lambda) \leqq 1-\frac{1}{\omega(\zeta)}, \quad \zeta \in \mathbb{T}
$$

For the other half, we use another collection of functions. For $\lambda \in \mathbb{D}$, let $G_{\lambda}$ be the function

$$
G_{\lambda}(z)=K_{\omega}(\lambda, \lambda)^{-\frac{1}{2}} K_{\omega}(z, \lambda), \quad z \in \mathbb{D},
$$

which has norm 1 in $P^{2}(\mathbb{D}, \omega)$. By the estimate of the kernel function of Theorem 5.1, and the well-known fact that $K_{\omega}(\lambda, \lambda) \rightarrow+\infty$ as $|\lambda| \rightarrow 1$ (this quantity represents the normsquared of the point evaluation functional, and the space $P^{2}(\mathbb{D}, \omega)$ contains the Hardy space $H^{2}(\mathbb{D})$ ), the function $G_{\lambda}$ tends to 0 uniformly off a fixed neighborhood of the point $\zeta$ as $\lambda$ approaches $\zeta \in \mathbb{T}$. In particular, the measure $\left|G_{\lambda}\right|^{2} \omega d \Sigma$ converges to a point mass at $\zeta$ as $\lambda \rightarrow \zeta$. Using the properties of the Bergman kernel for $P^{2}(\mathbb{D})$, we have the estimate

$$
K_{\omega}(\lambda, \lambda)=\left|G_{\lambda}(\lambda)\right|^{2}=\left|\int_{\mathbb{D}}(1-\lambda \bar{z})^{-2} G_{\lambda}(z) d \Sigma(z)\right|^{2} \leqq\left(1-|\lambda|^{2}\right)^{-2} \int_{\mathbb{D}}\left|G_{\lambda}(z)\right|^{2} d \Sigma(z),
$$

whereby in the limit,

$$
\int_{\mathbb{D}}\left|G_{\lambda}(z)\right|^{2} d \Sigma(z) \rightarrow \frac{1}{\omega(\zeta)} \quad \text { as } \lambda \rightarrow \zeta
$$

It follows that

$$
\begin{aligned}
1-\liminf _{\lambda \rightarrow \zeta} L_{\omega}(\lambda, \lambda) & =\limsup _{\lambda \rightarrow \zeta}\left(1-|\lambda|^{2} L_{\omega}(\lambda, \lambda)\right) \\
& =\limsup _{\lambda \rightarrow \zeta}\left(1-|\lambda|^{2}\right)^{2} K_{\omega}(\lambda, \lambda) \leqq 1-\frac{1}{\omega(\zeta)},
\end{aligned}
$$

and consequently,

$$
1-\frac{1}{\omega(\zeta)} \leqq \liminf _{\lambda \rightarrow \zeta} L_{\omega}(\lambda, \lambda), \quad \zeta \in \mathbb{T}
$$

It follows that $1 \leqq\left.\omega\right|_{\mathbb{T}}$, because a reproducing kernel is positive along the diagonal.

Remark 6.9. Let $\Gamma$ stand for the biharmonic Green function for $\mathbb{D}$, as given by the explicit formula (1.6). The-by now classical - factorization-type identity found in [8] for weights $\omega$ that reproduce for the origin reads 


$$
\begin{aligned}
\int_{\mathbb{D}}|f(z)|^{2} \omega(z) d \Sigma(z)= & \int_{\mathbb{D}}|f(z)|^{2} d \Sigma(z) \\
& +\int_{\mathbb{D} \times \mathbb{D}} \Gamma(z, \zeta)\left|f^{\prime}(z)\right|^{2} \Delta \omega(\zeta) d \Sigma(z) d \Sigma(\zeta), \quad f \in H^{2}(\mathbb{D}),
\end{aligned}
$$

and in view of the fact that $0<\Gamma(z, \zeta)$ on $\mathbb{D} \times \mathbb{D}$, and the logarithmic subharmonicity of $\omega$ which leads to $0 \leqq \Delta \omega$, we see that

$$
\int_{\mathbb{D}}|f(z)|^{2} d \Sigma(z) \leqq \int_{\mathbb{D}}|f(z)|^{2} \omega(z) d \Sigma(z), \quad f \in H^{2}(\mathbb{D}) .
$$

Suppose that $\omega$ extends to a continuous function on $\overline{\mathbb{D}}$. Then, by choosing the analytic function $f$ such that $|f|^{2} d \Sigma$ approximates a point mass at a point on the boundary $\mathbb{T}$, we have that $1 \leqq \omega(z)$ on $\mathbb{T}$.

The function $L_{\omega}$ is bounded and sesqui-holomorphic on $\mathbb{D}^{2}$-meaning that the function $L_{\omega}(z, \bar{\zeta})$ is a holomorphic function of two variables there-and hence it possesses radial boundary values almost everywhere on the torus $\mathbb{T}^{2}$, with respect to the usual area measure there. It follows that the kernels $K_{\omega}$ and $Q_{\omega}$, too, have radial boundary values almost everywhere on $\mathbb{T}^{2}$ : for $K_{\omega}$, we can use the formula defining $L_{\omega}$ in Theorem 6.3, and for $Q_{\omega}$, there is the identity of Proposition 2.3:

$$
Q_{\omega}(z, \zeta)=2 \operatorname{Re} K_{\omega}(z, \zeta)-1, \quad(z, \zeta) \in \mathbb{D}^{2} .
$$

The following result will be used later on in the proof of the positivity of the weighted biharmonic Green function $\Gamma_{\omega}$. For this reason, we specify explicitly all the requirements on the weight $\omega$.

Corollary 6.10. Let $\omega$ be a logarithmically subharmonic reproducing weight on $\mathbb{D}$, which is continuous on $\overline{\mathbb{D}}$. We then have the inequality (almost everywhere)

$$
Q_{\omega}(z, \zeta) \leqq-\left(\frac{1}{\omega(z)}+\frac{1}{\omega(\zeta)}\right) \frac{1}{|z-\zeta|^{2}}, \quad(z, \zeta) \in \mathbb{T} \times \mathbb{T} \backslash \delta(\mathbb{T}) .
$$

Proof. Since the kernel $L_{\omega}$ is reproducing for some space, we have

$$
\left|L_{\omega}(z, \zeta)\right| \leqq L_{\omega}(z, z)^{\frac{1}{2}} L_{\omega}(\zeta, \zeta)^{\frac{1}{2}}, \quad z, \zeta \in \mathbb{D}
$$

and in view of Theorem 6.8 and the geometric-arithmetic mean value inequality, we obtain, almost everywhere,

$$
\left|L_{\omega}(z, \zeta)\right| \leqq\left(1-\frac{1}{\omega(z)}\right)^{\frac{1}{2}}\left(1-\frac{1}{\omega(\zeta)}\right)^{\frac{1}{2}} \leqq 1-\frac{1}{2}\left(\frac{1}{\omega(z)}+\frac{1}{\omega(\zeta)}\right), \quad(z, \zeta) \in \mathbb{T}^{2}
$$

We write the equation for $K_{\omega}$ in terms of $L_{\omega}$ as

$$
K_{\omega}(z, \zeta)=\frac{1-z \bar{\zeta} L_{\omega}(z, \zeta)}{(1-z \bar{\zeta})^{2}}=\frac{1}{1-z \bar{\zeta}}+\frac{z \bar{\zeta}}{(1-z \bar{\zeta})^{2}}-\frac{z \bar{\zeta}}{(1-z \bar{\zeta})^{2}} L_{\omega}(z, \zeta), \quad(z, \zeta) \in \mathbb{D}^{2}
$$


where we notice the appearance of the Kœbe function

$$
\kappa(z)=\frac{z}{(1-z)^{2}}, \quad z \in \mathbb{D}
$$

which maps $\mathbb{D}$ onto the slit domain $\left.\mathbb{C} \backslash]-\infty,-\frac{1}{4}\right]$. Its boundary values are

$$
\kappa(z)=-\frac{1}{|1-z|^{2}}, \quad z \in \mathbb{T} \backslash\{1\}
$$

so that on $\mathbb{T}^{2} \backslash \delta(\mathbb{T}), K_{\omega}$ equals (almost everywhere)

$$
K_{\omega}(z, \zeta)=\frac{1}{1-z \bar{\zeta}}-\frac{1}{|z-\zeta|^{2}}-\frac{1}{|z-\zeta|^{2}} L_{\omega}(z, \zeta), \quad(z, \zeta) \in \mathbb{T}^{2} \backslash \delta(\mathbb{T}) .
$$

The first term on the right hand side has real part $\frac{1}{2}$. From the identity (6.15), the above representation formula for $K_{\omega}$, and (6.16), we see that (almost everywhere)

$$
\begin{aligned}
Q_{\omega}(z, \zeta) & =-\frac{2}{|z-\zeta|^{2}}-\frac{2}{|z-\zeta|^{2}} \operatorname{Re} L_{\omega}(z, \zeta) \leqq-\frac{2}{|z-\zeta|^{2}}+\frac{2}{|z-\zeta|^{2}}\left|L_{\omega}(z, \zeta)\right| \\
& =-\left(\frac{1}{\omega(z)}+\frac{1}{\omega(\zeta)}\right) \frac{1}{|z-\zeta|^{2}}, \quad(z, \zeta) \in \mathbb{T}^{2} \backslash \delta(\mathbb{T}),
\end{aligned}
$$

as asserted.

Remark 6.11. (a) Proposition 6.4 only uses the logarithmic subharmonicity of $\omega$, not the reproducing property.

(b) In the proof of Theorem 6.3, we appeal to Theorem 5.1 mainly for reasons of convenience of exposition. The use of it can be avoided entirely, and then one has a different proof of Theorem 5.1 from [22].

(c) It is possible to interpret the assertion of Theorem 6.8 as a statement about the asymptotic behavior of the matrix

$$
\left\{\hat{K}_{\omega}(j, k)\right\}_{j, k=0}^{\infty}
$$

For large indices, the increments of this matrix in the direction of the diagonal (but not necessarily on the diagonal) are asymptotically given in terms of the Fourier coefficients of the reciprocal weight $\left.\omega^{-1}\right|_{\mathbb{T}}$.

(d) If we assume more regularity of $\omega$, say $C^{\infty}$-smoothness on $\overline{\mathbb{D}}$, then the kernels $K_{\omega}$ and $Q_{\omega}$ are also much smoother, in fact, $C^{\infty}$-smooth on $\overline{\mathbb{D}} \times \overline{\mathbb{D}} \backslash \delta(\mathbb{T})$, so that the assertion of Corollary 6.10 is valid everywhere on $\mathbb{T}^{2} \backslash \delta(\mathbb{T})$.

(e) Suppose $\omega$ is $C^{\infty}$-smooth on $\overline{\mathbb{D}}$ and real-analytic near $\mathbb{T}$. It is a natural problem to ask under what additional assumptions the kernel $L_{\omega}$ becomes sesqui-holomorphic on 
$\overline{\mathbb{D}} \times \overline{\mathbb{D}}$. We recall that sesqui-holomorphic means that the function is holomorphic in the first variable, and anti-holomorphic in the second. One shows that unless $\Delta \log \omega=0$ along $\mathbb{T}$, the kernel $K_{\omega}$ necessarily develops a logarithmic singularity which prohibits such smoothness of $L_{\omega}$. An example of this phenomenon is $\omega(z)=\frac{3}{4}\left(1+|z|^{4}\right)$, with kernel

$$
K_{\omega}(z, \zeta)=\frac{2}{3}\left(\frac{1}{(1-z \bar{\zeta})^{2}}+\frac{1}{1-z \bar{\zeta}}-\frac{z \bar{\zeta}+\log (1-z \bar{\zeta})}{(z \bar{\zeta})^{2}}\right)
$$

\section{The weighted Hele-Shaw flow}

Here, we assume $\omega$ is a real-analytic weight on $\overline{\mathbb{D}}$, which is strictly positive there: $\omega(z)>0$ at all points of $\overline{\mathbb{D}}$. Moreover, $\omega$ is assumed logarithmically subharmonic, and reproducing for the origin:

$$
h(0)=\int_{\mathbb{D}} h(z) \omega(z) d \Sigma(z),
$$

for all bounded harmonic functions on $\mathbb{D}$. From [24], we have the following rather technical-looking result concerning the Hele-Shaw flow which is the result of injecting twodimensional fluid into the curved manifold obtained by supplying $\mathbb{D}$ with the Riemannian metric $d \mathbf{s}(z)=\sqrt{\omega(z)}|d z|$, with the origin as injection point. At time $t=1$, the whole unit disk is filled.

Theorem 7.1. For each $0<r \leqq 1$, there is a (unique) simply connected domain $D(r)$ contained in $\mathbb{D}$ and a conformal map $\phi_{r}: \mathbb{D} \rightarrow D(r)$ with the following properties:

(a) The boundary of $D(r), \partial D(r)$, is a real-analytic Jordan curve.

(b) Each $D(r)$ contains the origin and each $\phi_{r}$ preserves the origin.

(c) The domains $D(r)$ are increasing in $r$ with $D(1)=\mathbb{D}$, and their intersection is the point at the origin.

(d) The reproducing property

$$
r^{2} h(0)=\int_{\mathbb{D}(r)} h(z) \omega(z) d \Sigma(z)
$$

holds for all bounded harmonic functions $h$ on $D(r)$.

(e) The mapping $(r, z) \mapsto \phi_{r}(z)$ extends to a holomorphic function of two complex variables on a neighborhood of $(0,1] \times \overline{\mathbb{D}}$.

(f) For each $0<r^{\prime} \leqq 1$, there is a small open interval $J$ around it such that all the functions $\phi_{r}, r \in J$, extend as conformal maps to one and the same neighborhood of $\overline{\mathbb{D}}$.

(g) For each $0<r^{\prime}<1$, we have 


$$
\frac{1}{r^{2}-\left(r^{\prime}\right)^{2}} 1_{D(r) \backslash D\left(r^{\prime}\right)}(z) d \Sigma(z) \rightarrow \frac{d \varpi_{r^{\prime}}(z)}{\omega(z)}
$$

as $r \rightarrow r^{+}$, in the weak-star topology of the Borel measures. Here, $\varpi_{r}$ is harmonic measure on $\partial D(r)$ with respect to the origin.

(h) The evolution equation

$$
\frac{d \phi_{r}}{d r}(z)=r z \phi_{r}^{\prime}(z) \int_{\bar{T}} \frac{\zeta+z}{\zeta-z} \frac{d \sigma(\zeta)}{\omega\left(\phi_{r}(\zeta)\right)\left|\phi_{r}^{\prime}(\zeta)\right|^{2}}
$$

holds for all $0<r<1$ and $z \in \mathbb{D}$.

We will call the domain $D(r)$ an $\omega$-mean value disk of radius $r$. The reproducing property (d) above is the most fundamental; in fact, it uniquely determines the domain $D(r)$.

\section{Hadamard's variational formula}

The variational formula for the weighted biharmonic Green function. Let $\Gamma_{\omega, r}$ denote the weighted biharmonic Green function for the operator $\Delta \omega^{-1} \Delta$ on the Hele-Shaw flow domain $D(r)$ (see the previous section). Similarly, let $G_{r}$ be the classical Green function for $D(r)$, and let $H_{\omega, r}$ be the harmonic compensator corresponding to the weight $\omega$ and the domain $D(r)$. Since $\omega$ is real-analytic on $\overline{\mathbb{D}}$, we conclude using the elliptic regularity theorem of Morrey-Nirenberg [32] (for details, see Section 3) that the Green function $\Gamma_{\omega, r}$ extends real-analytically to a neighborhood of the set

$$
\bar{D}(r) \times \bar{D}(r) \backslash \delta(\bar{D}(r)),
$$

where

$$
\delta(\bar{D}(r))=\{(z, z): z \in \bar{D}(r)\}
$$

is the diagonal. In particular, for fixed $\zeta \in \bar{D}(r)$, the function $\Gamma_{\omega, r}$ solves the differential equation

$$
\Delta \omega^{-1} \Delta \Gamma_{\omega, r}(\cdot, \zeta)=\delta_{\zeta}
$$

on a neighborhood of $\bar{D}(r) \backslash\{\zeta\}$. It is intuitively clear that $\Gamma_{\omega, r}$ varies continuously with the parameter $r$; in fact, this can be made rigorous, for instance with the methods of Section 5. We shall derive a variational formula, originally found by Hadamard in 1908, which describes the development quantitatively [13], pp. 515-641. We follow the pattern from Hedenmalm's 1994 paper [19]. We consider two parameter values $r, r^{\prime}$ with $0<r<r^{\prime}<1$, 
and note that by (3.4) and (3.2), with obvious notations (for instance, $G_{r}$ is the Green function for $\Delta$ on $D(r)$ ),

$$
\Gamma_{\omega, r}(z, \zeta)=\int_{D(r)}\left[G_{r}(\xi, z)+H_{\omega, r}(\xi, z)\right]\left[G_{r^{\prime}}(\xi, \zeta)+H_{\omega, r^{\prime}}(\xi, \zeta)\right] \omega(\xi) d \Sigma(\xi)
$$

for $(z, \zeta) \in D(r) \times D(r)$, and for $(z, \zeta) \in D\left(r^{\prime}\right) \times D\left(r^{\prime}\right)$, we have

$$
\Gamma_{\omega, r^{\prime}}(z, \zeta)=\int_{D\left(r^{\prime}\right)}\left[G_{r}(\xi, z)+H_{\omega, r}(\xi, z)\right]\left[G_{r^{\prime}}(\xi, \zeta)+H_{\omega, r^{\prime}}(\xi, \zeta)\right] \omega(\xi) d \Sigma(\xi)
$$

As $r<r^{\prime}$, we have the inclusion $D(r) \subset D\left(r^{\prime}\right)$, so that forming the difference of the above relations, we obtain

$$
\begin{aligned}
& \text { (8.1) } \Gamma_{\omega, r^{\prime}}(z, \zeta)-\Gamma_{\omega, r}(z, \zeta) \\
& =\int_{D\left(r^{\prime}\right) \backslash D(r)}\left[G_{r}(\xi, z)+H_{\omega, r}(\xi, z)\right]\left[G_{r^{\prime}}(\xi, \zeta)+H_{\omega, r^{\prime}}(\xi, \zeta)\right] \omega(\xi) d \Sigma(\xi),
\end{aligned}
$$

whereby $(z, \zeta) \in D(r) \times D(r)$, but, if $r^{\prime}$ is sufficiently close to $r$, so that we can use the elliptic regularity, we can take $(z, \zeta) \in D\left(r^{\prime}\right) \times D\left(r^{\prime}\right)$. From Theorem 7.1, part (g), we know that as $r^{\prime} \rightarrow r$, the measure

$$
\frac{1}{\left(r^{\prime}\right)^{2}-r^{2}} 1_{D\left(r^{\prime}\right) \backslash D(r)}(z) \omega(z) d \Sigma(z)
$$

converges (in the weak-star topology) to the harmonic measure $\varpi_{r}$ for the point 0 in the domain $D(r)$, which is supported on $\partial D(r)$. Dividing both sides of (8.1) by $r^{\prime}-r$, and taking the limit as $r^{\prime} \rightarrow r$, we find that since the Green function $G_{r}$ vanishes when one of the variables is on the boundary $\partial D(r)$,

$$
\frac{d}{d r} \Gamma_{\omega, r}(z, \zeta)=2 r \int_{\partial D(r)} H_{\omega, r}(\xi, z) H_{\omega, r}(\xi, \zeta) d \varpi_{r}(\xi)
$$

Some further explanation is needed here. We need to know a priori that the kernels $\Gamma_{\omega, r}$, $H_{\omega, r}$, and $G_{r}$ depend fairly smoothly on the parameter $r$. It is convenient to use the conformal map $\phi_{r}: \mathbb{D} \rightarrow D(r)$ to pull back the situation to the unit disk. We then have the identity

$$
\Gamma_{\omega_{r}}(z, \zeta)=\Gamma_{\omega, r}\left(\phi_{r}(z), \phi_{r}(\zeta)\right), \quad(z, \zeta) \in \mathbb{D} \times \mathbb{D}
$$

whereby $\omega_{r}=\omega \circ \phi_{r}\left|\phi_{r}^{\prime}\right|^{2}$, and the left hand side expresses the Green function on the unit disk for the weighted biharmonic operator $\Delta \omega_{r}^{-1} \Delta$. By Theorem 7.1, the weight $\omega_{r}(z)$ is real-analytic (and strictly positive) in the coordinates $(z, r)$ on a neighborhood of $\overline{\mathbb{D}} \times] 0,1]$. The Green function $G_{r}$ is easily expressed in terms of the Green function $G=G_{\mathbb{D}}$ for the laplacian on the unit disk,

$$
G(z, \zeta)=G_{r}\left(\phi_{r}(z), \phi_{r}(\zeta)\right), \quad(z, \zeta) \in \mathbb{D} \times \mathbb{D}
$$


and this identity gives us fairly complete regularity information for $G_{r}$. As far as $\Gamma_{\omega_{r}}$ is concerned, we can turn to the proof of the elliptic regularity theorem of Morrey-Nirenberg [32], which gives us more quantitative information concerning the domain of convergence for the power series used to represent the real-anaytic functions. It can be shown that the kernel $\Gamma_{\omega_{r}}(z, \zeta)$ is real-analytic in the coordinates $(z, \zeta, r)$ on a neighborhood of the product set

$$
\left.\left.\left(\overline{\mathbb{D}}^{2} \backslash \delta(\overline{\mathbb{D}})\right) \times\right] 0,1\right]
$$

which then leads to the analogous information that $H_{\omega_{r}}(z, \zeta)$ is real-analytic in the coordinates $(z, \zeta, r)$ on a neighborhood of the slightly bigger set

$$
\left.\left.\left(\overline{\mathbb{D}}^{2} \backslash \delta(\mathbb{T})\right) \times\right] 0,1\right] .
$$

This justifies the limit process leading up to (8.2).

We want to turn the differential equation (8.2) into an integral equation. Note that when one of the variables $z, \zeta$ is on the boundary $\partial D(r)$, and the other is in the interior $D(r)$, the Green function for $\Delta \omega^{-1} \Delta$ vanishes: $\Gamma_{\omega, r}(z, \zeta)=0$. As we integrate (8.2) with respect to $r$, the following formula emerges:

$$
\Gamma_{\omega, r}(z, \zeta)=\int_{\max \{R(z), R(\zeta)\}}^{r} \int_{\partial D(\varrho)} H_{\omega, \varrho}(\xi, z) H_{\omega, \varrho}(\xi, \zeta) d \varpi_{\varrho}(\xi) 2 \varrho d \varrho
$$

for $(z, \zeta) \in D(r) \times D(r)$. Here, $R(z)$ stands for the parameter value of $\varrho$ for which the boundary of $D(\varrho)$ reaches the point $z$ :

$$
R(z)=\inf \{\varrho: z \in D(\varrho)\} .
$$

We know from Section 3 that if the Green function $\Gamma_{\omega, r}$ is positive on $D(r) \times D(r)$, then the corresponding harmonic compensator $H_{\omega, r}$ is positive on $\partial D(r) \times D(r)$, and since the latter is harmonic in the first variable, it is then positive throughout $D(r) \times D(r)$. Hadamard's variational formula (8.3) provides a kind of converse to the first implication: if all the harmonic compensators for the subdomains $D(\varrho), 0<\varrho<r$, are positive, then the Green function $\Gamma_{\omega, r}$ is positive.

The variational formula for the Green function for the laplacian. We turn to Hadamard's better-known variational formula for $G_{r}([33]$, p. 46), which has important applications to the theory of conformal mappings. Let $P_{r}$ be given by

$$
P_{r}(z, \zeta)=-\frac{1}{2} \partial_{n(\zeta)} G_{r}(z, \zeta), \quad(z, \zeta) \in D(r) \times \partial D(r)
$$

the normal derivative being taken with respect to the boundary $\partial D(r)$ in the interior direction. This function then serves as a Poisson kernel on $D(r)$. For instance, we have the identity

$$
d \varpi_{r}(z)=P_{r}(0, z) d \sigma(z), \quad z \in \partial D(r) .
$$

The variational formula states that 


$$
\frac{d}{d r} G_{r}(z, \zeta)=-2 r \int_{\partial D(r)} P_{r}(z, \xi) P_{r}(\zeta, \xi) \frac{d \varpi_{r}(\xi)}{\omega(\xi)}, \quad(z, \zeta) \in D(r) \times D(r)
$$

and in integral form, it becomes

$$
G_{r}(z, \zeta)=-\int_{\max \{R(z), R(\zeta)\}}^{r} \int_{\partial D(\varrho)} P_{\varrho}(z, \xi) P_{\varrho}(\zeta, \xi) \frac{d \varpi_{\varrho}(\xi)}{\omega(\xi)} 2 \varrho d \varrho
$$

for $(z, \zeta) \in D(r) \times D(r)$. We may combine this with equation (3.3), to get

$$
\begin{aligned}
H_{\omega, r}(\zeta, z)= & \int_{D(r) \max \{R(z), R(\eta)\}} \int_{\partial D(\varrho)}^{r} Q_{\omega, r}(\zeta, \eta) \\
& \times P_{\varrho}(z, \xi) P_{\varrho}(\eta, \xi) \frac{d \varpi_{\varrho}(\xi)}{\omega(\xi)} 2 \varrho d \varrho \omega(\eta) d \Sigma(\eta),
\end{aligned}
$$

which transforms to

$$
\begin{aligned}
H_{\omega, r}(\zeta, z)= & \int_{R(z)}^{r} \int_{\partial D(\varrho)} \int_{D(\varrho)} Q_{\omega, r}(\zeta, \eta) P_{\varrho}(\eta, \xi) \omega(\eta) d \Sigma(\eta) \\
& \times P_{\varrho}(z, \xi) \frac{d \varpi_{\varrho}(\xi)}{\omega(\xi)} 2 \varrho d \varrho, \quad(z, \zeta) \in D(r) \times D(r) .
\end{aligned}
$$

\section{Positivity of the weighted biharmonic Green function}

We continue the presentation from the previous section on Hadamard's variational formula. We recapture: the weight $\omega$ is real-analytic on $\overline{\mathbb{D}}$ and strictly positive there; we also suppose that $\log \omega$ is subharmonic on $\mathbb{D}$, and that $\omega$ reproduces for the origin:

$$
h(0)=\int_{\mathbb{D}} h(z) \omega(z) d \Sigma(z)
$$

holds for all bounded harmonic functions $h$ on $\mathbb{D}$. The domains $D(r)$, indexed by $r$, $0<r \leqq 1$, are the generalized "disks" about the origin of radius $r$ arising from the weighted Hele-Shaw flow, and these constitute a real-analytic continuous sequence of simply connected domains, whose boundaries are real-analytic Jordan curves. It is a consequence of formula $(8.5)$ that if we can prove that

$$
0 \leqq \int_{D(\varrho)} Q_{\omega, r}(\zeta, \eta) P_{\varrho}(\eta, \xi) \omega(\eta) d \Sigma(\eta), \quad(\xi, \zeta) \in \partial D(\varrho) \times D(r)
$$

whenever $0<\varrho<r \leqq 1$, then the harmonic compensator $H_{\omega, r}$ is positive on $D(r) \times D(r)$. In the above integral, the function $Q_{\omega}(\zeta, \cdot)$ is harmonic on $\bar{D}(r)$, and in particular, bounded there, and the Poisson kernel $P(\cdot, \xi)$ is area summable on $D(\varrho)$. We conclude that the integral in (9.1) makes sense.

We shall obtain the following result, which is equivalent to (9.1). 
Theorem 9.1. Fix $\varrho, r$ such that $0<\varrho<r \leqq 1$. Let $h$ be a positive harmonic function on $D(\varrho)$, and define

$$
h_{r}(z)=\int_{D(\varrho)} Q_{\omega, r}(z, \xi) h(\xi) \omega(\xi) d \Sigma(\xi), \quad z \in D(r)
$$

Then $h_{r}$ is positive on $D(r)$.

Proof. It suffices to obtain the result under the proviso that $h$ is harmonic and strictly positive on $\bar{D}(\varrho)$. Since $Q_{\omega, r}(0, \cdot)=r^{-2}$ (this is a consequence of the reproducing property of the flow domain $D(r)$ ), the value of the function $h_{r}$ at the center point 0 is

$$
h_{r}(0)=\frac{1}{r^{2}} \int_{D(\varrho)} h(\xi) \omega(\xi) d \Sigma(\xi)=\frac{\varrho^{2}}{r^{2}} h(0),
$$

which is positive. We split the proof into three parts.

Part 1: continuity of $h_{r}$ in $r$. The function $h_{r}$ is the orthogonal projection of $h 1_{D(\varrho)}$ interpreted to vanish on $D(r) \backslash D(\varrho)$ - onto the harmonic subspace $H L^{2}(D(r), \omega)$ in $L^{2}(D(r), \omega)$. From the smoothness of the harmonic compensator $H_{\omega, r}$ in the $r$ variable alluded to above, and the corresponding fact for the weighted harmonic Bergman kernel $Q_{\omega, r}$ as deduced from the identity

$$
Q_{\omega, r}(z, \zeta)=-\omega(z)^{-1} \Delta_{\zeta} H_{\omega, r}(z, \zeta)
$$

it is immediate that $h_{r}(z)$ is real-analytic in the coordinates $(z, r)$ on (a neighborhood of) the set

$$
\{(z, r): z \in \bar{D}(r), r \in] \varrho, 1]\} \cup\{(z, r): z \in D(r), r \in[\varrho, 1]\}
$$

We need to investigate the continuity of $h_{r}(z)$ near the left end-point $r=\varrho$. By the reproducing property of the flow domains with respect to the weight, we have that

$$
\int_{D(\varrho)} Q_{\omega, r}(z, \xi) \omega(\xi) d \Sigma(\xi)=\varrho^{2} Q_{\omega, r}(z, 0)=\frac{\varrho^{2}}{r^{2}}, \quad z \in D(r),
$$

and hence

$$
h_{r}(z)-\frac{r^{2}}{\varrho^{2}} h(z)=\int_{D(\varrho)} Q_{\omega, r}(z, \xi)(h(\xi)-h(z)) \omega(\xi) d \Sigma(\xi), \quad z \in D(r)
$$

provided $r$ is so close to $\varrho$ that $h$ is defined as a harmonic function on $D(r)$. We have that $h(\xi)-h(z)=O(|z-\xi|)$ for $z, \xi$ in some fixed neighborhood of $\bar{D}(\varrho)$, so that part of the singularity of the kernel $Q_{\omega, r}$ is neutralized by the appearance of this factor on the right hand side of (9.2). As before, let $\phi_{r}: \mathbb{D} \rightarrow D(r)$ be the Riemann map taking 0 onto 0 , and let $\omega_{r}$ stand for the pulled-back weight on the unit disk,

$$
\omega_{r}(z)=r^{-2} \omega \circ \phi_{r}(z)\left|\phi_{r}^{\prime}(z)\right|^{2},
$$


which is reproducing for the origin as well as logarithmically subharmonic. From the conformal invariance of the reproducing property of the weighted harmonic Bergman kernel, the following identity can be deduced:

$$
r^{2} Q_{\omega, r}\left(\phi_{r}(z), \phi_{r}(\zeta)\right)=Q_{\omega_{r}}(z, \zeta)=2 \operatorname{Re} K_{\omega_{r}}(z, \zeta)-1, \quad(z, \zeta) \in \mathbb{D} \times \mathbb{D}
$$

We apply Theorem 5.1 to $K_{\omega_{r}}$, and obtain as a result that

$$
r^{2}\left|Q_{\omega, r}\left(\phi_{r}(z), \phi_{r}(\zeta)\right)\right|=\left|Q_{\omega_{r}}(z, \zeta)\right| \leqq 1+\frac{4}{|1-z \bar{\zeta}|^{2}}, \quad(z, \zeta) \in \mathbb{D} \times \mathbb{D} .
$$

We rewrite (9.2) in terms of the variable $\zeta, \phi_{r}(\zeta)=\xi$, and get

$$
\begin{aligned}
& h_{r} \circ \phi_{r}(z)-\frac{r^{2}}{\varrho^{2}} h \circ \phi_{r}(z) \\
& \quad=r^{2} \int_{\phi_{r}^{-1}(D(\varrho))} Q_{\omega, r}\left(\phi_{r}(z), \phi_{r}(\zeta)\right)\left[h\left(\phi_{r}(\zeta)\right)-h\left(\phi_{r}(z)\right)\right] \omega_{r}(\zeta) d \Sigma(\zeta), \quad z \in \mathbb{D},
\end{aligned}
$$

where $\phi_{r}^{-1}(D(\varrho)) \subset \mathbb{D}$. Given the estimates mentioned previously, it is easily deduced from this identity that $h_{r} \circ \phi_{r} \rightarrow h \circ \phi_{\varrho}$ uniformly on $\mathbb{D}$ as $r \rightarrow \varrho$. In particular, since we assume $h$ to be strictly positive on $\bar{D}(\varrho)$, it follows that $h_{r} \circ \phi_{r}$ is uniformly (in $r$ ) strictly positive on $\overline{\mathbb{D}}$ for $r$ in some short interval $] \varrho, \varrho+\delta]$, with $0<\delta$.

Part 2: the derivative of $h_{r} \circ \phi_{r}$. The derivative of the composition $h_{r} \circ \phi_{r}$ with respect to the parameter $r$ is, by the chain rule,

$$
\frac{d}{d r} h_{r} \circ \phi_{r}(z)=\frac{\partial h_{r}}{\partial r} \circ \phi_{r}(z)+2 \operatorname{Re}\left(\frac{\partial h_{r}}{\partial z} \circ \phi_{r}(z) \frac{d \phi_{r}}{d r}(z)\right),
$$

where the partial derivatives with respect to $r$ and $z$ correspond to thinking of the function $h_{r}$ as a function of two variables: $h_{r}(z)=h(z, r)$. The derivative of $\phi_{r}$ with respect to $r$ is supplied by formula (h) of Theorem 7.1, which simplifies to

$$
\frac{d \phi_{r}}{d r}(z)=\frac{z}{r} \phi_{r}^{\prime}(z) \int_{\mathbb{T}} \frac{\zeta+z}{\zeta-z} \frac{d \sigma(\zeta)}{\omega_{r}(\zeta)}=\frac{z}{r} \phi_{r}^{\prime}(z) \mathfrak{H}_{+}\left[\frac{1}{\omega_{r}}\right](z), \quad z \in \mathbb{D},
$$

where the symbol $\mathfrak{H}_{+}$stands for the Herglotz transform. We would like to find a way to express the partial derivative $\partial_{r} h_{r}$. Let $r^{\prime}, \varrho<r^{\prime}<r$ be so close to $r$ that $h_{r^{\prime}}$ extends harmonically and boundedly to $D(r)$. Then, from the reproducing property of the weighted harmonic Bergman kernel, we have

$$
h_{r^{\prime}}(z)=\int_{D(r)} Q_{\omega, r}(z, \xi) h_{r^{\prime}}(\xi) \omega(\xi) d \Sigma(\xi), \quad z \in D(r)
$$

On the other hand, again by the reproducing property,

$$
\int_{D\left(r^{\prime}\right)} Q_{\omega, r}(z, \xi) Q_{\omega, r^{\prime}}(\xi, \zeta) \omega(\xi) d \Sigma(\xi)=Q_{\omega, r}(z, \zeta), \quad(z, \zeta) \in D(r) \times D\left(r^{\prime}\right)
$$


so that

$$
\begin{aligned}
\int_{D\left(r^{\prime}\right)} Q_{\omega, r}(z, \xi) h_{r^{\prime}}(\xi) \omega(\xi) d \Sigma(\xi) & \\
= & \int_{D\left(r^{\prime}\right)} Q_{\omega, r}(z, \xi) \int_{D(\varrho)} Q_{\omega, r^{\prime}}(\xi, \zeta) h(\zeta) \omega(\zeta) d \Sigma(\zeta) \omega(\xi) d \Sigma(\xi) \\
& =\int_{D(\varrho)} Q_{\omega, r}(z, \zeta) h(\zeta) \omega(\zeta) d \Sigma(\zeta)=h_{r}(z), \quad z \in D(r) .
\end{aligned}
$$

Forming the difference between (9.7) and (9.8), we obtain

$$
h_{r}(z)-h_{r^{\prime}}(z)=-\int_{D(r) \backslash D\left(r^{\prime}\right)} Q_{\omega, r}(z, \xi) h_{r^{\prime}}(\xi) \omega(\xi) d \Sigma(\xi), \quad z \in D(r) .
$$

In view of the observation made following Theorem 7.1, to the effect that the measure

$$
\frac{1}{r^{2}-\left(r^{\prime}\right)^{2}} 1_{D(r) \backslash D\left(r^{\prime}\right)}(z) \omega(z) d \Sigma(z)
$$

converges weak-star to the harmonic measure $\varpi_{r}$ on the boundary for domain $D(r)$ with respect to the interior point 0 as $r^{\prime} \rightarrow r$, it follows from (9.9) that

$$
\frac{\partial h_{r}}{\partial r}(z)=-2 r \int_{\partial D(r)} Q_{\omega, r}(z, \xi) h_{r}(\xi) d \varpi_{r}(\xi), \quad z \in D(r) .
$$

Shifting the coordinates back to the unit disk, we obtain, keeping in mind (9.3),

$$
\frac{\partial h_{r}}{\partial r} \circ \phi_{r}(z)=-\frac{2}{r} \int_{\mathbb{T}} Q_{\omega_{r}}(z, \zeta) h_{r} \circ \phi_{r}(\zeta) d \sigma(\zeta), \quad z \in \mathbb{D} .
$$

By the Poisson integral formula for harmonic functions in $\mathbb{D}$, we have the representation

$$
h_{r} \circ \phi_{r}(z)=\int_{\mathbb{V}} \frac{1-|z|^{2}}{|1-z \bar{\zeta}|^{2}} h_{r} \circ \phi_{r}(\zeta) d \sigma(\zeta), \quad z \in \mathbb{D},
$$

which after an application of one of the two Wirtinger differential operators leads to

$$
\phi_{r}^{\prime}(z) \frac{\partial h_{r}}{\partial z} \circ \phi_{r}(z)=\int_{\mathbb{T}} \frac{\bar{\zeta}}{(1-z \bar{\zeta})^{2}} h_{r} \circ \phi_{r}(\zeta) d \sigma(\zeta), \quad z \in \mathbb{D} .
$$

In view of (9.6), (9.10), and (9.11), the identity (9.5) becomes

$$
\begin{aligned}
& \frac{d}{d r} h_{r} \circ \phi_{r}(z) \\
& \quad=\frac{2}{r} \int_{\mathbb{T}}\left\{\operatorname{Re}\left(\mathfrak{Y}_{+}\left[\frac{1}{\omega_{r}}\right](z) \frac{z \bar{\zeta}}{(1-z \bar{\zeta})^{2}}\right)-Q_{\omega_{r}}(z, \zeta)\right\} h_{r} \circ \phi_{r}(\zeta) d \sigma(\zeta), \quad z \in \mathbb{D} .
\end{aligned}
$$


As in the proof of Corollary 6.10, we notice the appearance of the Kœbe function. Suppose for the moment that for some value of the parameter $r, \varrho<r \leqq 1$, the real-analytic function $\left.h_{r} \circ \phi_{r}\right|_{\mathbb{T}}$ vanishes along with its (tangential) derivative at some point $z_{1} \in \mathbb{T}$. Then $h_{r} \circ \phi_{r}(z)=O\left(\left|z-z_{1}\right|^{2}\right)$ as $z$ approaches $z_{1}$ along $\mathbb{T}$, which counterbalances the singularities of the Kœbe function and the weighted harmonic Bergman kernel, as estimated by (9.4), at least when $z \in \mathbb{D}$ approaches the boundary point $z_{1}$ radially. Taking into account the well-known boundary behavior of the Kobe function, we obtain in the limit that (the real part of the Herglotz transform is the Poisson integral, with well-known boundary values)

$$
\frac{d}{d r} h_{r} \circ \phi_{r}\left(z_{1}\right)=-\frac{2}{r} \int_{\mathbb{T}}\left\{\frac{1}{\omega_{r}\left(z_{1}\right)} \frac{1}{\left|\zeta-z_{1}\right|^{2}}+Q_{\omega_{r}}\left(z_{1}, \zeta\right)\right\} h_{r} \circ \phi_{r}(\zeta) d \sigma(\zeta) .
$$

If, in addition, $0 \leqq h_{r} \circ \phi_{r}$ on $\overline{\mathbb{D}}$, well, then, by invoking Corollary 6.10 , which states that

$$
Q_{\omega_{r}}\left(z_{1}, \zeta\right) \leqq-\left(\frac{1}{\omega_{r}\left(z_{1}\right)}+\frac{1}{\omega_{r}(\zeta)}\right) \frac{1}{\left|\zeta-z_{1}\right|^{2}}, \quad \zeta \in \mathbb{T} \backslash\left\{z_{1}\right\}
$$

we can assert that

$$
0<\frac{2}{r} \int_{\mathbb{V}} \frac{1}{\omega_{r}(\zeta)} \frac{1}{\left|\zeta-z_{1}\right|^{2}} h_{r} \circ \phi_{r}(\zeta) d \sigma(\zeta) \leqq \frac{d}{d r} h_{r} \circ \phi_{r}\left(z_{1}\right)
$$

The leftmost inequality holds because $h_{r} \circ \phi_{r}$ cannot vanish identically - after all, we know that $0<h_{r}(0)=h_{r} \circ \phi_{r}(0)$.

Part 3: the finishing argument. Consider the function

$$
\mathfrak{h}(r)=\min \left\{h_{r}(z): z \in \bar{D}(r)\right\}=\min \left\{h_{r} \circ \phi_{r}(z): z \in \overline{\mathbb{D}}\right\}, \quad \varrho<r \leqq 1,
$$

which, by the results of Part 1 , extends continuously to the interval $[\varrho, 1]$, and is positive at the left end-point: $0<\mathfrak{h}(\varrho)$. We shall demonstrate that $0<\mathfrak{h}(r)$ holds for all $r \in[\varrho, 1]$, which is actually slightly stronger than what is needed. We argue by contradiction, and assume $\mathfrak{h}(r) \leqq 0$ for some $r \in] \varrho, 1[$. Forming the infimum over all such $r$, we find a parameter value $\left.r_{1} \in\right] \varrho, 1\left[\right.$ with $\mathfrak{h}\left(r_{1}\right)=0$, such that $0<\mathfrak{h}(r)$ holds for all $r \in\left[\varrho, r_{1}[\right.$. By the maximum principle, this means that there exists a point $z_{1} \in \mathbb{T}$, such that $h_{r_{1}} \circ \phi_{r_{1}}\left(z_{1}\right)=0$, and that $0 \leqq h_{r_{1}} \circ \phi_{r_{1}}$ elsewhere on $\overline{\mathbb{D}}$. The point $z_{1}$ is precisely of the type considered in Part 2, so that by (9.14),

$$
0<\left.\frac{d}{d r} h_{r} \circ \phi_{r}\left(z_{1}\right)\right|_{r=r_{1}} .
$$

We immediately see that $h_{r} \circ \phi_{r}\left(z_{1}\right)<0$ for $r, \varrho<r<r_{1}$, sufficiently close to $r_{1}$, and hence $\mathfrak{h}(r)<0$ for such $r$. This contradicts the minimality of $r_{1}$, and completes the proof.

From the previous section on Hadamard's variational formula, we then have the following corollary. 
Corollary 9.2. Fix $r$ with $0<r \leqq 1$. Then both $H_{\omega, r}$ and $\Gamma_{\omega, r}$ are positive on $D(r) \times D(r)$.

In the corollary, we need in fact not the entire assumption that $\omega$ is logarithmically subharmonic throughout $\Omega$ : it can be weakened to requiring $\log \omega$ to be subharmonic on $D(r)$. This leads immediately to the following result, where $\omega$ is a weight on the unit disk.

Corollary 9.3. Suppose $\omega$ is a logarithmically subharmonic and reproducing (for the origin) weight on $\mathbb{D}$, real-analytic on $\overline{\mathbb{D}}$, and strictly positive on $\overline{\mathbb{D}}$ as well. Then the weighted biharmonic Green function $\Gamma_{\omega}$ is positive on $\mathbb{D} \times \mathbb{D}$.

In view of the sections on approximation of weights and Green functions (Sections 4 and 5, and more to the point, Theorems 4.1 and 5.2), we can remove the regularity assumptions in the above corollary.

Corollary 9.4. Suppose $\omega$ is a logarithmically subharmonic and reproducing (for the origin) weight on $\mathbb{D}$. Then the weighted biharmonic Green function $\Gamma_{\omega}$ is positive on $\mathbb{D} \times \mathbb{D}$.

The above corollary was obtained earlier by Shimorin [43] in the special case of a radial weight; see also Hedenmalm [19], [21].

\section{Applications to the Bergman spaces}

Applications to the Bergman spaces $\boldsymbol{A}^{\boldsymbol{p}}(\mathbb{D})$. The study of the kernel function in the context of the Bergman spaces was initiated by Stefan Bergman [6]. However, in the first couple of attempts toward a factorization theory for the Bergman spaces - by Charles Horowitz [26], [27], and Boris Korenblum [28] — it played a subordinate rôle, if used at all. The kernel function later reappeared in the work of Hedenmalm [15]. Given a zero sequence $A$ for the Hilbert Bergman space $A^{2}(\mathbb{D})$ on the unit disk (which for simplicity avoids the origin), he considered the invariant subspace $M_{A}$ of all functions in $A^{2}(\mathbb{D})$ that vanish on $A$ (counting multiplicities), and formed the function

$$
\varphi_{A}(z)=K_{A}(0,0)^{-\frac{1}{2}} K_{A}(z, 0), \quad z \in \mathbb{D},
$$

where $K_{A}$ denotes the reproducing kernel for $M_{A}$. The function $\varphi_{A}$ has norm 1 in $A^{2}(\mathbb{D})$, and has largest value in modulus at the origin among all functions in the closed unit ball of $M_{A}$; for this reason, such functions are sometimes called extremal functions. Hedenmalm showed that $\varphi_{A}$ is an expansive multiplier, that is,

$$
\|f\|_{A^{2}} \leqq\left\|\varphi_{A} f\right\|_{A^{2}}, \quad f \in A^{2}(\mathbb{D}) .
$$

For infinite zero sequences $A$, it may happen that the right hand side attains the value $+\infty$. Furthermore, the function $\varphi_{A}$ has no extraneous zeros in $\mathbb{D}$, and it is a contractive divisor,

$$
\left\|f / \varphi_{A}\right\|_{A^{2}} \leqq\|f\|_{A^{2}}, \quad f \in M_{A}
$$


In [7], [8], the quartet Duren-Khavinson-Shapiro-Sundberg generalized Hedenmalm's results to the Bergman spaces $A^{p}(\mathbb{D}), 0<p<+\infty$. In the context of $A^{2}(\mathbb{D})$, the main idea is to write the kernel function $K_{A}$ in the form

$$
K_{A}(z, \zeta)=b_{A}(z) \bar{b}_{A}(\zeta) K_{\left|b_{A}\right|^{2}}(z, \zeta), \quad(z, \zeta) \in \mathbb{D} \times \mathbb{D}
$$

where $b_{A}$ is the Blaschke product for $A$, assuming the sequence $A$ meets the Blaschke condition. For general $p, 0<p<+\infty$, we then set

$$
\varphi_{A}(z)=K_{\left|b_{A}\right|^{p}}(0,0)^{-\frac{1}{p}} b_{A}(z) K_{\left|b_{A}\right|^{p}}(z, 0)^{\frac{2}{p}}, \quad z \in \mathbb{D},
$$

where as it happens, the weighted Bergman kernel function fails to have zeros, so that it is all right to take fractional powers of it. These functions $\varphi_{A}$ can be defined for all zero sequences, not just the for ones that satisfy the Blaschke condition, and have factorization properties analogous to what was the case for $p=2$. An important observation is that the weight $\left|\varphi_{A}\right|^{p}$ is a logarithmically subharmonic and reproducing for the origin. Multiplication by $\varphi_{A}$ is an isometry $P^{p}\left(\mathbb{D},\left|\varphi_{A}\right|^{p}\right) \rightarrow A^{p}(\mathbb{D})$ (evident notation), so that the question whether for two zero sequences $A$ and $B$,

$$
\left\|\varphi_{A} f\right\|_{A^{p}} \leqq\left\|\varphi_{B} f\right\|_{A^{p}}
$$

holds for all polynomials $f$, becomes a matter of whether the injection mapping

$$
P^{p}\left(\mathbb{D},\left|\varphi_{B}\right|^{p}\right) \rightarrow P^{p}\left(\mathbb{D},\left|\varphi_{A}\right|^{p}\right)
$$

is a contraction.

We have the following theorem.

Theorem 10.1. Let $A$ and $B$ be two zero sequences for $A^{p}(\mathbb{D})$, such that $A$ is contained in $B$. Then

$$
\left\|\varphi_{A} f\right\|_{A^{p}} \leqq\left\|\varphi_{B} f\right\|_{A^{p}}, \quad f \in A^{p}(\mathbb{D}) .
$$

Proof. For finite sequences $A$ and $B$, the functions $\varphi_{A}$ and $\varphi_{B}$ are holomorphic in a neighborhood of $\overline{\mathbb{D}}$, and we consider the function $\Phi_{B, A}$ which solves the boundary value problem

$$
\begin{aligned}
\Delta \Phi_{B, A}(z) & =\left|\varphi_{B}(z)\right|^{p}-\left|\varphi_{A}(z)\right|^{p}, & & z \in \mathbb{D}, \\
\Phi_{B, A}(z) & =0, & & z \in \mathbb{T} .
\end{aligned}
$$

From an application of Green's formula, as in [15], [7], [8], we see that the fact that the right hand side-the function $\left|\varphi_{B}\right|^{p}-\left|\varphi_{A}\right|^{p}$ - annihilates the harmonic functions in $L^{2}(\mathbb{D})$ translates to the additional boundary data

$$
\partial_{n(z)} \Phi_{B, A}(z)=0, \quad z \in \mathbb{T} .
$$

Dividing the differential equation by $\left|\varphi_{A}(z)\right|^{2}$, and then afterward applying another laplacian, we find that it solves 


$$
\Delta \frac{1}{\left|\varphi_{A}(z)\right|^{p}} \Delta \Phi_{B, A}(z)=\Delta\left|\frac{\varphi_{B}(z)}{\varphi_{A}(z)}\right|^{p}, \quad z \in \mathbb{D},
$$

which is positive on $\mathbb{D}$. In view of the given boundary data, we may write the function $\Phi_{B, A}$ as an integral in terms of the weighted biharmonic Green function $\Gamma_{\left|\varphi_{A}\right|^{p}}$ :

$$
\Phi_{B, A}(z)=\int_{\mathbb{D}} \Gamma_{\left|\varphi_{A}\right|^{p}}(z, \zeta) \Delta_{\zeta}\left|\frac{\varphi_{B}(\zeta)}{\varphi_{A}(\zeta)}\right|^{p} d \Sigma(\zeta), \quad z \in \mathbb{D}
$$

which is then positive. The importance of the potential function $\Phi_{B, A}$ comes from the fact that Green's formula yields the identity

$$
\left\|\varphi_{B} f\right\|_{A^{p}}^{p}-\left\|\varphi_{A} f\right\|_{A^{p}}^{p}=\int_{\mathbb{D}} \Phi_{B, A}(z) \Delta_{z}|f(z)|^{p} d \Sigma(z), \quad z \in \mathbb{D}
$$

for polynomials $f$, which yields the desired inequality in this case, because we can approximate functions in $A^{p}(\mathbb{D})$ by polynomials, and because the functions $\varphi_{A}$ and $\varphi_{B}$ are bounded on $\mathbb{D}$. Setting $g=\varphi_{B} f$, we conclude that

$$
\left\|\frac{\varphi_{A}}{\varphi_{B}} g\right\|_{A^{p}} \leqq\|g\|_{A^{p}}
$$

for all $g \in A^{p}(\mathbb{D})$ that vanish on $B$. Now let $A$ and $B$ be arbitrary zero sequences, and form finite subsequences $A^{\prime} \subset A$ and $B^{\prime} \subset B$, with $A^{\prime} \subset B^{\prime}$. Then the above inequality holds with $A$ and $B$ replaced by $A^{\prime}$ and $B^{\prime}$, respectively, and we apply it to $g$ vanishing on $B$. Letting $A^{\prime}$ grow up to $A$, and $B^{\prime}$ up to $B, \varphi_{A^{\prime}} \rightarrow \varphi_{A}$ and $\varphi_{B^{\prime}} \rightarrow \varphi_{B}$ in $A^{p}(\mathbb{D})$, and Fatou's lemma delivers the above inequality for arbitrary $A$ and $B$, which implies the assertion of the theorem.

Corollary $10.2(p=2)$. Let $A$ be and $B$ be two zero sequences for $A^{2}(\mathbb{D})$, and suppose that $B \backslash A$ consists of a single point $\alpha \in \mathbb{D}$. Then the quotient $\varphi_{\alpha}=\varphi_{B} / \varphi_{A}$ is a bounded holomorphic function on $\mathbb{D}$, and it only vanishes at the point $\alpha$ in $\mathbb{D}$. Moreover, if $b_{\alpha}$ is the Blaschke factor corresponding to the point $B \backslash A$, then $1 \leqq\left|\varphi_{\alpha} / b_{\alpha}\right|$ holds throughout $\mathbb{D}$. In particular, $\varphi_{\alpha}(\mathbb{D})$ covers the whole disk $\mathbb{D}$.

Proof. The function $\varphi_{\alpha}$ is given by the formula

$$
\varphi_{\alpha}(z)=\left(1-K_{\left|\varphi_{A}\right|^{2}}(\alpha, \alpha)^{-1}\right)^{-\frac{1}{2}}\left(1-\frac{K_{\left|\varphi_{A}\right|^{2}}(z, \alpha)}{K_{\left|\varphi_{A}\right|^{2}}(\alpha, \alpha)}\right), \quad z \in \mathbb{D},
$$

which, in view of Theorem 5.1, shows that $\varphi_{\alpha}$ is bounded on $\mathbb{D}$, at least for $\alpha \in \mathbb{D} \backslash\{0\}$. A closer analysis of what happens as $\alpha \rightarrow 0$ reveals that it is bounded also for $\alpha=0$. Using a peaking function argument as in [15], we obtain $1 \leqq\left|\varphi_{\alpha} / b_{\alpha}\right|$ on $\mathbb{D}$, as a consequence of Theorem 10.1, at least for finite sequences $A$. Approximating general zero sequences by finite ones, the assertion follows for general $A$.

The following answers a question raised by Korenblum. 
Corollary 10.3. Let $B$ be a zero sequence for $A^{p}(\mathbb{D})$ and $M$ an invariant subspace in $A^{p}(\mathbb{D})$. Suppose $M$ has index 1 , that is, the dimension of the quotient space $M / S M$ is 1 , where $S$ is the operator of multiplication by $z$. Then if $M_{B} \subset M$, the subspace $M$ is of the form $M=M_{A}$, for some smaller zero sequence $A$.

Proof. We follow the scheme from Hedenmalm [17]. Let $A$ be the sequence of common zeros (counting multiplicities) of the functions in $M$. We are to show that $M=M_{A}$. According to Theorem 5.2 and Proposition 5.4 of Aleman-Richter-Sundberg [3], it suffices to obtain that $\varphi_{A} \in M$, because $\varphi_{A}$ generates $M_{A}$ as an invariant subspace. From the assumption $M_{B} \subset M$, we have that $\varphi_{B} \in M$. Then, because $M$ has index 1, we may divide out superfluous zeros in $\varphi_{B}$, one by one, and remain in $M$. So, if $q$ is a polynomial whose zeros constitute a finite subset of $B \backslash A$, the function $\varphi_{B} / q$ is also in $M$. Let $A^{\prime}$ be a finite subsequence of $A$, and $B^{\prime}$ one of $B$, with $A^{\prime} \subset B^{\prime}$ and $\left(B^{\prime} \backslash A^{\prime}\right) \cap A=\emptyset$. Then the function $\varphi_{B^{\prime}} / \varphi_{A^{\prime}}$ can be factored as an invertible element of $H^{\infty}(\mathbb{D})$ times a polynomial $q$ with zeros at $B^{\prime} \backslash A^{\prime}$, and hence

$$
\varphi_{A^{\prime}, B^{\prime}, B}=\frac{\varphi_{A^{\prime}}}{\varphi_{B^{\prime}}} \varphi_{B} \in M
$$

Meanwhile, by (10.1),

$$
\left\|\frac{\varphi_{A^{\prime}}}{\varphi_{B^{\prime}}} \varphi_{B}\right\|_{A^{p}} \leqq\left\|\varphi_{B}\right\|_{A^{p}}=1
$$

The function $\varphi_{A^{\prime}, B^{\prime}, B}$ tends to $\varphi_{A}$ as $A^{\prime}$ grows to $A$ and $B^{\prime}$ grows to $B$, and the limit element $\varphi_{A}$ has norm 1, so that nothing is lost in Fatou's lemma. This means that $\varphi_{A^{\prime}, B^{\prime}, B}$ tends to $\varphi_{A}$ in norm, and the conclusion $\varphi_{A} \in M$ follows. The proof is complete.

Applications to weighted Bergman spaces. The main theorem enables us to develop a factorization theory for the spaces $P^{2}(\mathbb{D}, \omega)$, where the weight $\omega$ is assumed to be logarithmically subharmonic and reproducing for the origin. We say that a function $\varphi \in P^{2}(\mathbb{D}, \omega)$ is a $P^{2}(\mathbb{D}, \omega)$-inner function provided that the weight $|\varphi|^{2} \omega$ is reproducing for the origin.

Theorem 10.4. Assume $\omega$ is logarithmically subharmonic and reproducing for the origin. Let $\varphi \in P^{2}(\mathbb{D}, \omega)$ be a $P^{2}(\mathbb{D}, \omega)$-inner function. Then $\|f\|_{\omega} \leqq\|\varphi f\|_{\omega}$ for all polynomials $f$. In fact, we have the norm identity

$$
\|\varphi f\|_{\omega}^{2}=\|f\|_{\omega}^{2}+\int_{\mathbb{D} \times \mathbb{D}} \Gamma_{\omega}(z, \zeta)\left|\varphi^{\prime}(z)\right|^{2}\left|f^{\prime}(\zeta)\right|^{2} d \Sigma(z) d \Sigma(\zeta), \quad f \in P^{2}\left(\mathbb{D},|\varphi|^{2} \omega\right)
$$

Proof. The proof is really in the same vein as that of, for instance, Theorem 10.1. A slightly different approach is needed, though, because of the lack of smoothness assumption on the weight, in which case Definition 3.1 is used to define the Green function $\Gamma_{\omega}$. The calculations are analogous to the ones used in [18] (see also [3]), and therefore omitted.

A variant of the above runs as follows.

Theorem 10.5. Assume that $\omega$ and $\omega^{\prime}$ are two $C^{\infty}$-smooth weights on $\overline{\mathbb{D}}$ that reproduce for the origin. Suppose that in addition, $\omega$ is logarithmically subharmonic and that the quotient $\omega^{\prime} / \omega$ is subharmonic. Then $\|f\|_{\omega} \leqq\|f\|_{\omega^{\prime}}$ holds for all $f \in A^{2}(\mathbb{D})$. 
We remark that because of the regularity assumptions on $\omega$ and $\omega^{\prime}$, the spaces $P^{2}(\mathbb{D}, \omega)$ and $P^{2}\left(\mathbb{D}, \omega^{\prime}\right)$ coincide with $A^{2}(\mathbb{D})$ in the above theorem (the norms are different, though).

By the general theory of reproducing kernel functions, as found in Saitoh's book [37], Theorem 10.5 leads to the following conclusion.

Corollary 10.6. Under the assumptions of Theorem 10.5, the difference $K_{\omega}-K_{\omega^{\prime}}$ is a reproducing kernel on $\mathbb{D} \times \mathbb{D}$. In other words,

$$
\frac{L_{\omega^{\prime}}(z, \zeta)-L_{\omega}(z, \zeta)}{(1-z \bar{\zeta})^{2}}
$$

is a reproducing kernel on $\mathbb{D} \times \mathbb{D}$.

\section{Directions for further research}

The main result of this paper, the positivity of the weighted biharmonic Green function $\Gamma_{\omega}$ for logarithmically subharmonic reproducing weights $\omega$, was conjectured by Hedenmalm in 1992. Partial results in this direction were found by Hedenmalm [16], [19], [20], [21], by Sergei Shimorin [39], [40], [41], [43], and by Engliš [9], [10]. As a matter of fact, the variational technique used in [21] was an inspiration for this work, although the context is different.

It appears likely that the strong maximum principle suggested in the introduction should be true. We formulate this as a conjecture. The normal derivative is in the interior direction.

Conjecture 11.1. Let $\omega$ be a logarithmically subharmonic reproducing weight on $\mathbb{D}$, which is $C^{\infty}$-smooth on $\overline{\mathbb{D}}$. Let u be a $C^{\infty}$-smooth real-valued function on $\overline{\mathbb{D}}$. We then have the maximum principle

$$
\left.\left\{\left.\Delta \omega^{-1} \Delta u\right|_{\mathbb{D}} \leqq 0,\left.u\right|_{\mathbb{T}} \leqq 0,\left.\frac{\partial u}{\partial n}\right|_{\mathbb{T}} \leqq 0\right\} \Rightarrow u\right|_{\mathbb{D}} \leqq 0 .
$$

In terms of the Green function $\Gamma_{\omega}$, what is required is that

$$
\frac{\partial}{\partial n(z)} \frac{1}{\omega(z)} \Delta_{z} \Gamma_{\omega}(z, \zeta)=-2 P(z, \zeta)+\frac{\partial}{\partial n(z)} H_{\omega}(z, \zeta) \leqq 0, \quad(z, \zeta) \in \mathbb{T} \times \mathbb{D} .
$$

In the special case $\omega(z) \equiv 1$, an explicit calculation yields

$$
\frac{\partial}{\partial n(z)} \Delta_{z} \Gamma(z, \zeta)=-2\left(1-|\zeta|^{2}\right)\left(\frac{1}{|1-z \bar{\zeta}|^{2}}+\operatorname{Re} \frac{z \bar{\zeta}}{(1-z \bar{\zeta})^{2}}\right)<0, \quad(z, \zeta) \in \mathbb{T} \times \mathbb{D}
$$

In [42], Shimorin showed that one-point zero divisors are univalent functions - just like the individual Blaschke factors - in the weighted spaces $P^{2}(\mathbb{D}, \omega)$, where $\omega$ is radial 
and logarithmically subharmonic. He also showed that, modulo some regularity, a univalent one-point zero divisor is automatically an expansive multiplier; see also [4]. We believe that the following is true.

Conjecture 11.2. Let $\omega$ be a logarithmically subharmonic reproducing weight on $\mathbb{D}$. Then, for each $\alpha \in \mathbb{D} \backslash\{0\}$, the one-point zero divisor in $P^{2}(\mathbb{D}, \omega)$,

$$
\varphi_{\alpha}(z)=\left(1-K_{\omega}(\alpha, \alpha)^{-1}\right)^{-\frac{1}{2}}\left(1-\frac{K_{\omega}(z, \alpha)}{K_{\omega}(\alpha, \alpha)}\right), \quad z \in \mathbb{D}
$$

is univalent and maps $\mathbb{D}$ onto a star-shaped domain. Moreover, $\left|\varphi_{\alpha}(z)\right|<3$ holds for all $z \in \mathbb{D}$.

In fact, if the extended maximum principle holds, as formulated in Conjecture 11.1, then each one-point zero divisor $\varphi_{\alpha}$ is univalent and maps $\mathbb{D}$ onto a star-shaped domain, which by Corollary 10.2 contains $\mathbb{D}$. Assuming this for the moment, the geometry of the "corona" $\phi_{\alpha}(\mathbb{D}) \backslash \mathbb{D}$ should contain information about how close the space $P^{2}(\mathbb{D}, \omega)$ is to the limit case $H^{2}(\mathbb{D})$. We wish to point out that the focus on one-point zero divisors is not as special as it may seem: each zero-divisor can be written as a product of one-point zero divisors, where each factor is a divisor with respect to a weight that is the original weight times the modulus-squared of product of the previous factors.

We turn to the connection between Conjectures 11.1 and 11.2. We can write $\left|\varphi_{\alpha}\right|^{2}$ as an integral,

$$
\left|\varphi_{\alpha}(z)\right|^{2}=1+\int_{\mathbb{D}}\left[G(z, \zeta)+H_{\omega}(z, \zeta)\right]\left|\varphi_{\alpha}^{\prime}(\zeta)\right|^{2} d \Sigma(\zeta), \quad z \in \mathbb{D}
$$

and assuming that Conjecture 11.1 holds, we obtain that on the unit circle $\mathbb{T},\left|\varphi_{\alpha}\right|^{2}$ increases in the outward normal direction. Then, assuming that $\omega$ is real-analytic near $\mathbb{T}$, an application of the Cauchy-Riemann equations to the locally defined function $\log \varphi_{\alpha}$ shows that the argument of $\varphi_{\alpha}\left(e^{i \theta}\right)$ increases with $\theta$. The case of more general weights is obtained by approximation.

A way to reach more detailed information about $\Gamma_{\omega}$ is to try to dissect the kernel function $K_{\omega}$ further than we did back in Section 6. We should like to obtain further structural information about the kernel $L_{\omega}$.

In view of Corollary 10.6, one may ask the question whether the difference $L_{\omega^{\prime}}-L_{\omega}$ is also a reproducing kernel on $\mathbb{D} \times \mathbb{D}$, under the assumptions of Theorem 10.5. It turns out that it is not so, even if we replace the subharmonicity of $\omega^{\prime} / \omega$ with logarithmic subharmonicity.

Example. If $L_{\omega^{\prime}}-L_{\omega}$ were a reproducing kernel function for all logarithmically subharmonic reproducing weights $\omega, \omega^{\prime}$ with $\omega^{\prime} / \omega$ logarithmically subharmonic, subject to the condition that both weights are $C^{\infty}$-smooth on $\overline{\mathbb{D}}$, then it would also be so, by a dilation argument, for the radial weights

$$
\omega(z)=\omega_{\alpha}(z)=(1-\alpha)\left(1-|z|^{2}\right)^{-\alpha}, \quad z \in \mathbb{D},
$$


and

$$
\omega^{\prime}(z)=\omega_{\beta}(z)=(1-\beta)\left(1-|z|^{2}\right)^{-\beta}, \quad z \in \mathbb{D}
$$

which are less regular near the boundary, where the parameters $\alpha, \beta$ range over $0<\alpha<\beta<1$. The corresponding Bergman kernels are well-known, and we only write down the formula for $\alpha$ :

$$
K_{\omega_{\alpha}}(z, \zeta)=(1-z \bar{\zeta})^{-2+\alpha}, \quad(z, \zeta) \in \mathbb{D} \times \mathbb{D}
$$

We calculate that along the diagonal,

$$
L_{\omega_{\beta}}(z, z)-L_{\omega_{\alpha}}(z, z)=\frac{\left(1-|z|^{2}\right)^{\alpha}-\left(1-|z|^{2}\right)^{\beta}}{|z|^{2}}, \quad z \in \mathbb{D},
$$

which is positive, but fails to be subharmonic, which is necessary for it to be the restriction to the diagonal of a reproducing kernel function.

The above example, however, leaves open the possibility that at least one of the expressions

$$
\frac{L_{\omega^{\prime}}(z, \zeta)-L_{\omega}(z, \zeta)}{1-z \bar{\zeta}}, \quad(z, \zeta) \in \mathbb{D} \times \mathbb{D}
$$

and

$$
\frac{L_{\omega^{\prime}}(z, \zeta)-L_{\omega}(z, \zeta)}{1-z \bar{\zeta} L_{\omega}(z, \zeta)}, \quad(z, \zeta) \in \mathbb{D} \times \mathbb{D}
$$

might be a reproducing kernel. We note that if the second one is a reproducing kernel, then so is the first one, because it can be written as a product of the second one and the reproducing kernel $J_{\omega}$ of Theorem 6.2.

Creeping flow. The slow motion of a viscous incompressible fluid (with small Reynolds number), squeezed in between two parallel walls of constant infinitesimal width, is known as creeping flow. The motion of the fluid is governed by a real-valued potential function $\Psi$, which satisfies the biharmonic equation $\Delta^{2} \Psi=0$; the velocity of the fluid is given by the expression

$$
\left(\frac{\partial \Psi}{\partial y},-\frac{\partial \Psi}{\partial x}\right)
$$

which constitutes a vector perpendicular to the gradient $\nabla \Psi$. Consequently, the level curves of $\Psi$ are the flow lines of the creeping flow. Let the potential function $\Psi$ equal the biharmonic Green function $\Gamma_{\Omega}(\cdot, \zeta)$, for a fixed $\zeta \in \Omega$, where $\Omega$ is a bounded planar domain $\Omega$ with smooth boundary. The corresponding flow then involves a kind of "torque" applied at $\zeta$, and friction at the boundary $\partial \Omega$, so that the velocity vanishes there. There appears a 
main swirl (vortex) centered at a point near $\zeta$, and it is of interest to know whether there are any other smaller swirls (eddies) located further away from $\zeta$. Apparently, this is the same as asking whether the Green function $\Gamma_{\Omega}(\cdot, \zeta)$ has more than one local extreme point in $\Omega$. Let us consider the weighted biharmonic Green function $\Gamma_{\omega}$ for a logarithmically subharmonic reproducing weight $\omega$ on the unit disk $\mathbb{D}$. We may think of it as corresponding to a creeping flow in curved space. By our main theorem, the function $\Gamma_{\omega}$ is positive. This suggests that for fixed $\zeta \in \mathbb{D}$, the flow has no eddy immediately near $\mathbb{T}$. The question arises: is there only one swirl in this situation? For the weight $\omega(z) \equiv 1$, it is indeed so [12].

Acknowledgements. The first-named author, Håkan Hedenmalm, wishes to thank Peter Larkin Duren for his hospitality and encouragement during the summer of 1998 in Ann Arbor, Michigan. He thanks Björn Gustafsson for helpful remarks concerning hyperbolic geometry and Hele-Shaw flow. He also thanks the Erwin Schrödinger Institute in Vienna and in particular its director Peter Michor for offering a congenial atmosphere in June, 1999. The third-named author, Sergei Shimorin, thanks the Swedish Natural Science Research Council (NFR) for its support under grant M-AA/AS 12325-300. He also thanks the Russian Fund for Fundamental Studies for its support under grant 96-01-00693. We thank Lennart Carleson and Boris Korenblum for constructive criticism.

\section{References}

[1] L. Ahlfors, L. Sario, Riemann surfaces, Princeton Math. Ser. 26, Princeton University Press, Princeton, NJ, 1960.

[2] A. Aleman, The multiplication operators on Hilbert spaces of analytic functions, Habilitationsschrift, Fernuniversität Hagen, 1993.

[3] A. Aleman, S. Richter, C. Sundberg, Beurling's theorem for the Bergman space, Acta Math. 177 (1996), 275310 .

[4] A. Aleman, H. Hedenmalm, S. Richter, C. Sundberg, Curious properties of canonical divisors in weighted Bergman spaces, Proceedings of the conference in honor of B. Ya. Levin, Tel-Aviv University 1997, to appear.

[5] M. Berger, B. Gostiaux, Differential geometry: manifolds, curves, and surfaces, Grad. Texts Math. 115, Springer-Verlag, New York-Berlin 1988.

[6] S. Bergman, The kernel function and conformal mapping (second, revised edition), Math. Surv. Monogr. 5, American Mathematical Society, Providence, RI, 1970.

[7] P. L. Duren, D. Khavinson, H. S. Shapiro, C. Sundberg, Contractive zero-divisors in Bergman spaces, Pacific J. Math. 157 (1993), 37-56.

[8] P. L. Duren, D. Khavinson, H. S. Shapiro, C. Sundberg, Invariant subspaces and the biharmonic equation, Michigan Math. J. 41 (1994), 247-259.

[9] M. Engliš, A Loewner-type lemma for weighted biharmonic operators, Pacific J. Math. 179 (1997), $343-353$.

[10] M. Engliš, Weighted biharmonic Green functions for rational weights, Glasgow Math. J. 41 (1999), $239-269$.

[11] P. R. Garabedian, A partial differential equation arising in conformal mapping, Pacific J. Math. 1 (1951), 485-524.

[12] H.-C. Grunau, G. Sweers, Nonexistence of local minima of supersolutions for the circular clamped plate, Pac. J. Math. 198 (2001), 437-442.

[13] J. Hadamard, Euvres de Jacques Hadamard, Vols. 1-4, Editions du Centre National de la Recherche Scientifique, Paris 1968.

[14] V. P. Havin, N. K. Nikolski, ed., Linear and complex analysis, problem book 3, Part II, Lect. Notes Math. 1574, Springer-Verlag, Berlin 1994.

[15] H. Hedenmalm, A factorization theorem for square area-integrable analytic functions, J. reine angew. Math. 422 (1991), 45-68.

[16] H. Hedenmalm, A factorization theorem for a weighted Bergman space, St. Petersburg Math. J. 4 (1992), $163-174$. 
[17] H. Hedenmalm, An invariant subspace of the Bergman space having the codimension two property, J. reine angew. Math. 443 (1993), 1-9.

[18] H. Hedenmalm, A factoring theorem for the Bergman space, Bull. London Math. Soc. 26 (1994), $113-126$.

[19] H. Hedenmalm, A computation of the Green function for the weighted biharmonic operators $\Delta|z|^{-2 \alpha} \Delta$, with $\alpha>-1$, Duke Math. J. 75 (1994), 51-78.

[20] H. Hedenmalm, Open problems in the function theory of the Bergman space, Festschrift in honour of Lennart Carleson and Yngve Domar (Uppsala 1993), 153-169, Acta Univ. Upsaliensis Skr. Uppsala Univ. C Organ. Hist. 58, Uppsala Univ., Uppsala 1995.

[21] H. Hedenmalm, Boundary value problems for weighted biharmonic operators, St. Petersburg Math. J. 8 (1997), 661-674.

[22] H. Hedenmalm, An off-diagonal estimate of Bergman kernels, J. Math. Pures Appl. 79 (2000), $163-172$.

[23] H. Hedenmalm, S. Jakobsson, S. Shimorin, A maximum principle à la Hadamard for biharmonic operators with applications to the Bergman spaces, C. R. Acad. Sci. Paris Sér. I Math. 328 (1999), no. 11, 973-978.

[24] H. Hedenmalm, S. Shimorin, Hele-Shaw flow on hyperbolic surfaces, J. Math. Pures Appl. 81 (2002), $187-222$.

[25] H. Hedenmalm, $K$. Zhu, On the failure of optimal factorization for certain weighted Bergman spaces, Complex Var. Th. Appl. 19 (1992), 165-176.

[26] C. Horowitz, Zeros of functions in the Bergman spaces, Duke Math. J. 41 (1974), 693-710.

[27] C. Horowitz, Factorization theorems for functions in the Bergman spaces, Duke Math. J. 44 (1977), $201-213$.

[28] B. Korenblum, An extension of the Nevanlinna theory, Acta Math. 135 (1975), 187-219.

[29] B. Korenblum, A maximum principle for the Bergman space, Publ. Mat. 35 (1991), 479-486.

[30] C. Lewner, On generation of solutions of the biharmonic equation in the plane by conformal mappings, Pacific J. Math. 3 (1953), 417-436.

[31] V. A. Malyshev, Hadamard's conjecture and estimates of the Green function, St. Petersburg Math. J. 4 (1993), 633-666.

[32] C. B. Morrey, L. Nirenberg, On the analyticity of the solutions of linear elliptic systems of partial differential equations, Comm. Pure Appl. Math. 10 (1957), 271-290.

[33] Z. Nehari, Conformal mapping (Reprinting of the 1952 McGraw-Hill edition), Dover Publications, Inc., New York 1975.

[34] L. Nirenberg, Remarks on strongly elliptic partial differential equations, Comm. Pure Appl. Math. 8 (1955), 649-675.

[35] Ch. Pommerenke, Boundary behaviour of conformal maps, Grundl. Math. Wiss. 299, Springer-Verlag, Berlin 1992.

[36] S. Richter, A representation theorem for cyclic analytic two-isometries, Trans. Amer. Math. Soc. 328 (1991), 325-349.

[37] S. Saitoh, Theory of reproducing kernels and its applications, Pitman Res. Notes Math. Ser. 189, Longman Scientific \& Technical, Harlow 1988.

[38] H. S. Shapiro, The Schwarz function and its generalization to higher dimensions, Univ. Arkansas Lect. Notes Math. Sci. 9, Wiley-Interscience, John Wiley \& Sons, Inc., New York 1992.

[39] S. M. Shimorin, Factorization of analytic functions in weighted Bergman spaces, St. Petersburg Math. J. 5 (1994), 1005-1022.

[40] S. M. Shimorin, On a family of conformally invariant operators, St. Petersburg Math. J. 7 (1996), $287-306$.

[41] S. M. Shimorin, The Green function for the weighted biharmonic operator $\Delta\left(1-|z|^{2}\right)^{-\alpha} \Delta$ and the factorization of analytic functions (Russian), Zap. Nauchn. Sem. S.-Peterburg. Otdel. Mat. Inst. Steklov. (POMI) 222 (1995), Issled. po Linein. Oper. i Teor. Funktsii 23, 203-221.

[42] S. M. Shimorin, Single point extremal functions in weighted Bergman spaces, Nonlinear boundary-value problems and some questions of function theory, J. Math. Sci. 80 (1996), 2349-2356.

[43] S. M. Shimorin, The Green functions for weighted biharmonic operators of the form $\Delta w^{-1} \Delta$ in the unit disk, Some questions of mathematical physics and function theory, J. Math. Sci. (New York) 92 (1998), 44044411.

Department of Mathematics, Lund University, P.O. Box 118, 22100 Lund, Sweden

e-mail: haakan@maths.lth.se

e-mail: stefanj@maths.lth.se

e-mail: shimorin@maths.lth.se 